Pure and Applied Mathematics Quarterly

Volume 5, Number 1

(Special Issue: In honor of

Jean-Pierre Serre, Part 2 of 2$)$

$227-253,2009$

\title{
Stratification of Hurwitz Spaces by Closed Modular Subvarieties
}

\author{
Anna Cadoret and Akio Tamagawa
}

\begin{abstract}
In this paper we explain how an arithmetical invariant for Gcover - we call the base invariant - provides a stratification of Hurwitz moduli spaces of G-covers of the projective line by closed subvarieties. This stratification geometrically encodes arithmetical properties of Hurwitz spaces.
\end{abstract}

Keywords: moduli spaces, curves with group action, inverse Galois theory.

\section{Preliminaries}

Let $S$ be a connected scheme. An $S$-curve of genus $g$ is a smooth, proper, geometrically connected $S$-scheme of dimension 1 whose geometric fibers have genus $g$.

Given a finite group $G$ of order prime to the characteristics of $S$, an $S$-G-curve with group $G$ is a pair $(X, \alpha)$, where $X$ is an $S$-curve and $\alpha: G \hookrightarrow \operatorname{Aut}_{S}(X)$ is a group monomorphism. Two $S$-G-curves $\left(X_{i}, \alpha_{i}\right), i=1,2$ with the same group $G$ are $S$-G-isomorphic if there exists an $S$-scheme isomorphism $u: X_{1} \rightarrow X_{2}$ such that $u \alpha_{1}(g) u^{-1}=\alpha_{2}(g), g \in G$. An $S$-G-cover with group $G$ is a pair $(f: X \rightarrow Y, \alpha)$, where $f: X \rightarrow Y$ is a Galois cover of $S$-curves (that is a finite flat surjective morphism with generically separable geometric fibers over $S$ such that $\operatorname{Aut}_{Y}(X)$ acts transitively on the fibers) and $\alpha: G \stackrel{\sim}{\rightarrow} \operatorname{Aut}_{Y}(X)$ is a group isomorphism. Two $S$-G-covers $\left(X_{i} \rightarrow Y, \alpha_{i}\right), i=1,2$ of a given $S$-curve 
$Y \rightarrow S$ with the same group $G$ are $S$-G-isomorphic if there exists a $Y$-scheme isomorphism $u: X_{1} \rightarrow X_{2}$ such that $u \alpha_{1}(g) u^{-1}=\alpha_{2}(g), g \in G$. Two $S$-Gcovers $\left(X_{i} \rightarrow Y_{i}, \alpha_{i}\right), i=1,2$ with the same group $G$ are weakly $S$-G-isomorphic if there exists an $S$-scheme isomorphism $v: Y_{1} \rightarrow Y_{2}$ such that the $S$-G-covers $\left(v \circ f_{1}: X_{1} \rightarrow Y_{2}, \alpha_{1}\right)$ and $\left(f_{2}: X_{2} \rightarrow Y_{2}, \alpha_{2}\right)$ are $S$-G-isomorphic. The groupoid of $S$-G-curves with group $G$ and $S$-G-isomorphisms is then equivalent to the groupoid of $S$-G-covers with group $G$ and weak $S$-G-isomorphisms. In the following, we will drop the $\alpha$ in our notation though it remains part of the data. In particular, the set of G-covers $X \rightarrow \mathbb{P}_{S}^{1}$ of the projective line over $S$ with group $G$ can be equipped with two structures of groupoids: the G-structure, where isomorphisms are $S$-G-isomorphisms and the $G / P G L_{2}$-structure, where isomorphisms are weak $S$-G-isomorphisms.

Given a field $k$, we will write $\Gamma_{k}$ for its absolute Galois group and we will always assume that a compatible system $\left(\zeta_{n}\right)_{n \geq 1}$ of primitive roots of unity is given in the algebraic closure $\bar{k}$ of $k$ (that is, $\zeta_{n m}^{n}=\zeta_{m}, n, m \geq 1$ ).

Let $k$ be an algebraically closed field of characteristic not dividing $|G|$ and let $Y \rightarrow k$ be a $k$-curve. Then two classical invariants can be associated to a G-cover $f: X \rightarrow Y$ defined over $k$ with group $G$ : the ramification divisor $\mathbf{t} \subset Y$ and the inertia canonical invariant $\mathbf{C}=\left(C_{t}\right)_{t \in \mathbf{t}} \cdot{ }^{1}$ Writing $\mathcal{C}(G)$ for the set of all conjugacy classes of $G$ and $\mathcal{C}(G)^{*}$ for the set of all nontrivial conjugacy classes of $G$, the inertia canonical invariant $\mathbf{C}$ of a G-cover $f$ with group $G$ can be regarded as an element of the submonoid $\mathrm{R}_{+}^{*}(G):=\mathbb{N}^{\left(\mathcal{C}(G)^{*}\right)}$ of the free abelian monoid $\mathrm{R}_{+}(G):=\mathbb{N}^{(\mathcal{C}(G))}$ of all maps $n: \mathcal{C}(G) \rightarrow \mathbb{N}$. More explicitly, $\mathbf{C}=\left(C_{t}\right)_{t \in \mathbf{t}}$ is identified with $\sum_{C \in \mathcal{C}(G)} n(C) C$, where $n(C)$ is the number of $t \in \mathbf{t}$ with $C_{t}=C$. The monoid $\mathrm{R}_{+}(G)$ can be endowed with a degree epimorphism $\operatorname{deg}: \mathrm{R}_{+}(G) \rightarrow \mathbb{N}$ sending $\mathbf{C}=\sum_{C \in \mathcal{C}(G)} n(C) C$ to $\sum_{C \in \mathcal{C}(G)} n(C)$. Hence, when $\mathbf{C}$ is the inertia canonical invariant of a G-cover $f, \operatorname{deg}(\mathbf{C})$ is just the degree $r$ of the ramification divisor $\mathbf{t}$ of $f$. For the general theory of G-covers over arbitrary schemes, we refer to $[1, \S 3]$. In particular, given a connected $\mathbb{Z}\left[\frac{1}{|G|}\right]$-scheme $S$, the inertia canonical

\footnotetext{
${ }^{1}$ Recall that $\mathbf{C}=\left(C_{t}\right)_{t \in \mathbf{t}}$ is defined as follows. For each $t \in \mathbf{t}$, choose a place $P_{t}$ in $k(X)$ above $t$ and let $I_{P_{t}}$ be the corresponding inertia group, which is cyclic of order $e_{t}$. Any uniformizing parameter $u$ of $P_{t}$ induces a well-defined (independent of the uniformizing parameter $u$ ) group monomorphism $\phi_{P_{t}}: I_{P_{t}} \hookrightarrow k^{\times}, \omega \rightarrow \frac{\omega(u)}{u} \bmod P_{t}$. The element $\omega_{P_{t}}:=\alpha\left(\phi_{P_{t}}^{-1}\left(\zeta_{e_{t}}\right)\right) \in G$ is called the distinguished generator of $I_{P_{t}}$. The set of all $\omega_{P_{t}}$ for places $P_{t}$ above $t$ is a full conjugacy class $C_{t}$ in $G$. In $[1, \S 2.2 .1]$ an equivalent definition of the inertia canonical invariant is given in terms of "holonomy".
} 
invariant $\mathbf{C}_{s}$ of the geometric fiber of an $S$-G-cover $f: X \rightarrow Y$ does not depend (up to $\Gamma_{\mathbb{Q}^{-}}$-conjugate) on the geometric point $s \in S$. Hence, we denote it by $\mathbf{C}$ and call it the inertia canonical invariant of $f: X \rightarrow Y$.

Given a finite group $G$, an integer $r \geq 3$ and a degree $r$ element $\mathbf{C} \in \mathrm{R}_{+}^{*}(G)$, we will denote the groupoids of G-covers and $\mathrm{G} / \mathrm{PGL}_{2}$-covers of the projective line over $S$ with group $G$ and inertia canonical invariant $\mathbf{C}$ by $\mathcal{H}_{\mathrm{G}}(\mathbf{C})(S)$ and $\mathcal{H}_{\mathrm{G} / \mathrm{PGL}_{2}}(\mathbf{C})(S)$ respectively. Given furthermore an integer $g \geq 0$, we will denote the groupoid of genus $g S$-G-curves with group $G$ whose resulting G-cover has inertia canonical invariant $\mathbf{C}$ by $\mathcal{H}_{g, G, \mathbf{C}}(S) . S \mapsto \mathcal{H}_{\mathrm{G}}(\mathbf{C})(S)$ and $S \mapsto \mathcal{H}_{g, G, \mathbf{C}}(S)$ are Deligne-Mumford stacks over $\mathbb{Z}_{\mathbf{C}}\left[\frac{1}{G]}\right]$ and their coarse moduli spaces are schemes that we will denote by $\mathrm{H}_{G}(\mathbf{C})$ and $\mathrm{H}_{g, G, \mathbf{C}}$ respectively. (See [16] and [1] for more details). Here, $\mathbb{Z}_{\mathbf{C}}$ is the ring of integers of a certain number field $\mathbb{Q}_{\mathbf{C}}$. (See section 2.1 for the definition of $\left.\mathbb{Q}_{\mathbf{C}}\right)$. On the other hand, $S \mapsto \mathcal{H}_{\mathrm{G} / \mathrm{PGL}_{2}}(\mathbf{C})(S)$ defines a prestack but not a stack (since descent data are not effective in general) but its associated stack is $\mathcal{H}_{g(\mathbf{C}), G, \mathbf{C}}$. (See section 2.1 for the definition of $g(\mathbf{C})$ ).

In section 2.2, we show that the quotient of $H_{\mathrm{G}}(\mathbf{C})$ under the natural action of $\mathrm{PGL}_{2}$ exists over $\mathbb{Q}_{\mathbf{C}}$ and yields a coarse moduli scheme $H_{\mathrm{G} / \mathrm{PGL}_{2}}(\mathbf{C})$ for the prestack $\mathcal{H}_{\mathrm{G} / \mathrm{PGL}_{2}}(\mathbf{C})$ over $\mathbb{Q}_{\mathbf{C}}$; we denote the quotient morphism by $\pi: H_{\mathrm{G}}(\mathbf{C}) \rightarrow H_{\mathrm{G} / \mathrm{PGL}_{2}}(\mathbf{C})$. We also explain that $\mathrm{H}_{g(\mathbf{C}), G, \mathbf{C}}$ is, as well, a coarse moduli scheme for the prestack $\mathcal{H}_{\mathrm{G} / \mathrm{PGL}_{2}}(\mathbf{C})$ over $\mathbb{Z}_{\mathbf{C}}\left[\frac{1}{|G|}\right]$ (hence we will denote it by $H_{\mathrm{G} / \mathrm{PGL}_{2}}(\mathbf{C})$ ). The aim of this paper is to give a stratification of (the generic fiber of) these moduli schemes by closed modular subvarieties.

Fix a field $k$ of characteristic 0 . Given a G-cover $f: X \rightarrow \mathbb{P} \frac{1}{k}$ define the base group $E_{f}$ of $f$ to be the stabilizer of the G-isomorphism class of $f$ under $\mathrm{PGL}_{2}{ }^{2}$ and the base invariant of $f$ to be the conjugacy class $\mathcal{E}_{f}$ of $E_{f}$ in $\mathrm{PGL}_{2}$; the latter is an invariant of the $\mathrm{G} / \mathrm{PGL}_{2}$-isomorphism class of $f$. Note that $E_{f}$ stabilizes the ramification divisor $\mathbf{t}$ of $f$ and hence, provided $r:=\operatorname{deg}(\mathbf{t}) \geq 3$, it is contained in the permutation group $\mathfrak{S}(\mathbf{t})$. In particular, for a given $r \geq 3$, there are only finitely many possible values for the base invariant. This results from the following classification result for finite subgroups of $\mathrm{PGL}_{2}(\bar{k})[14]$, which asserts in particular that, for a given $r \geq 3$, there are only finitely many conjugacy classes of finite subgroups of $\mathrm{PGL}_{2}(\bar{k})$ contained in a copy of the symmetric group $\mathcal{S}_{r}$.

\footnotetext{
${ }^{2}$ Note that, by definition, the centralizer of $G$ in $\operatorname{Aut}(X)$ is then an extension of $E_{f}$ by the center $Z(G)$ of $G$ and that $E_{f}$ acts trivially on $Z(G)$.
} 
Lemma 1.1. Any finite subgroup of $\mathrm{PGL}_{2}(\bar{k})$ is conjugate to one of the following:

- $C_{n}=\left\{\left(\begin{array}{cc}\zeta_{n}^{r} & 0 \\ 0 & 1\end{array}\right), r=0, \ldots, n-1\right\}$, where $\zeta_{n}$ is a primitive $n$th root of unity, $n \geq 1$;

- $D_{2 n}=\left\{\left(\begin{array}{cc}\zeta_{n}^{r} & 0 \\ 0 & 1\end{array}\right),\left(\begin{array}{cc}0 & \zeta_{n}^{r} \\ 1 & 0\end{array}\right), r=0, \ldots, n-1\right\}$, where $\zeta_{n}$ is a primitive $n$th root of unity, $n \geq 2$;

$-\mathcal{A}_{4}=\left\{\left(\begin{array}{cc} \pm 1 & 0 \\ 0 & 1\end{array}\right),\left(\begin{array}{ll}0 & \pm 1 \\ 1 & 0\end{array}\right),\left(\begin{array}{cc}i^{\nu} & i^{\nu} \\ 1 & -1\end{array}\right),\left(\begin{array}{cc}i^{\nu} & -i^{\nu} \\ 1 & 1\end{array}\right),\left(\begin{array}{cc}1 & i^{\nu} \\ 1 & -i^{\nu}\end{array}\right),\left(\begin{array}{cc}-1 & -i^{\nu} \\ 1 & -i^{\nu}\end{array}\right), \nu=1,3\right\} ;$

- $\mathcal{S}_{4}=\left\{\left(\begin{array}{cc}i^{\nu} & 0 \\ 0 & 1\end{array}\right),\left(\begin{array}{ll}0 & i^{\nu} \\ 1 & 0\end{array}\right),\left(\begin{array}{ll}i^{\nu} & -i^{\nu+\nu^{\prime}} \\ 1 & i^{\nu^{\prime}}\end{array}\right), \nu, \nu^{\prime}=0,1,2,3\right\}$;

- $\mathcal{A}_{5}=\left\{\left(\begin{array}{cc}\zeta^{r} & 0 \\ 0 & 1\end{array}\right),\left(\begin{array}{cc}0 & \zeta^{r} \\ 1 & 0\end{array}\right),\left(\begin{array}{cc}\zeta^{r} \omega & \zeta^{r-s} \\ 1 & -\zeta^{s} \omega\end{array}\right),\left(\begin{array}{cc}\zeta^{r} \bar{\omega} & \zeta^{r-s} \\ 1 & -\zeta^{-s} \bar{\omega}\end{array}\right), r, s=0,1,2,3,4\right\}$, where $\omega=\frac{-1+\sqrt{5}}{2}, \bar{\omega}=\frac{-1-\sqrt{5}}{2}$ and $\zeta$ is a primitive 5 th root of unity.

One can define a partial order $\subset$ on conjugacy classes of finite subgroups of $\mathrm{PGL}_{2}(\bar{k})$ by $\mathcal{E}_{1} \subset \mathcal{E}_{2}$ if and only if $E_{1} \subset E_{2}$ for some representatives $E_{1}, E_{2}$ of $\mathcal{E}_{1}, \mathcal{E}_{2}$ or, equivalently, there exists a monomorphism $i: E_{1} \hookrightarrow E_{2}$ (indeed, by lemma 1.1, two finite subgroups of $\mathrm{PGL}_{2}(\bar{k})$ are isomorphic if and only if they are conjugate in $\mathrm{PGL}_{2}(\bar{k})$ and note that giving a monomorphism $E_{1} \hookrightarrow E_{2}$ is equivalent to giving an isomorphism between $E_{1}$ and a subgroup of $E_{2}$ ). Now, given a conjugacy class $\mathcal{E}$ of finite subgroups of $\mathrm{PGL}_{2}(\bar{k})$ denote by $H_{\mathrm{G}}(\mathbf{C}, \supset \mathcal{E})(k)$ (resp. $\left.H_{\mathrm{G}}(\mathbf{C}, \mathcal{E})(k)\right)$ the subset of $k$-rational points of $H_{\mathrm{G}}(\mathbf{C})$ corresponding to G-covers $f$ with base invariant $\mathcal{E}_{f} \supset \mathcal{E}$ (resp. with base invariant $\mathcal{E}_{f}=\mathcal{E}$ ). We define similarly $H_{\mathrm{G} / \mathrm{PGL}_{2}}(\mathbf{C}, \supset \mathcal{E})(k)$ (resp. $\left.H_{\mathrm{G}_{\mathrm{PGL}}}(\mathbf{C}, \mathcal{E})(k)\right)$.

In [3], constructing a cohomological obstruction for the lifting of $k$-rational points from $H_{\mathrm{G} / \mathrm{PGL}_{2}}(\mathbf{C})$ to $H_{\mathrm{G}}(\mathbf{C})$, one shows that, for any nontrivial conjugacy class $\mathcal{E}$, there exists an integer $1 \leq d(\mathcal{E}) \leq 6$ depending only on $\mathcal{E}$ and not on $r$ such that the image of the natural map

$$
\bigcup_{[l: k] \leq d(\mathcal{E})} H_{\mathrm{G}}(\mathbf{C}, \mathcal{E})(l) \rightarrow H_{\mathrm{G} / \mathrm{PGL}_{2}}(\mathbf{C}, \mathcal{E})(\bar{k})
$$

contains $H_{\mathrm{G} / \mathrm{PGL}_{2}}(\mathbf{C}, \mathcal{E})(k)$. So, the subsets $H_{\mathrm{G}}(\mathbf{C}, \supset \mathcal{E})\left(\right.$ resp. $\left.H_{\mathrm{G}_{\mathrm{PG}} \mathrm{PG}_{2}}(\mathbf{C}, \supset \mathcal{E})\right)$ of points in $H_{\mathrm{G}}(\mathbf{C})$ (resp. in $H_{\mathrm{G} / \mathrm{PGL}_{2}}(\mathbf{C})$ ) corresponding to G-covers $f$ with base invariant $\mathcal{E}_{f} \supset \mathcal{E}$ have particularly "good" arithmetical properties. They actually are geometrical objects, as follows. 
Theorem 1.2. For any conjugacy class $\mathcal{E}$ of finite subgroups of $\mathrm{PGL}_{2}(\overline{\mathbb{Q}})$, the set $H_{\mathrm{G}}(\mathbf{C}, \supset \mathcal{E})$ is closed in $H_{\mathrm{G}}(\mathbf{C})$. In particular, $H_{\mathrm{G}}(\mathbf{C}, \supset \mathcal{E})$ can be equipped with a unique structure of reduced closed subvariety defined over $\mathbb{Q}_{\mathbf{C}}$.

To prove theorem 1.2 , it is enough to show that the inverse image of $H_{\mathrm{G}}(\mathbf{C}, \supset$ $\mathcal{E})$ is closed in $H_{\mathrm{G}}(\mathbf{C}) \times_{\mathbb{Q}_{\mathbf{C}}} \overline{\mathbb{Q}}$, that is, this inverse image coincides with the underlying set of a closed subvariety $H_{\mathrm{G}}(\mathbf{C}, \supset \mathcal{E})$ of $H_{\mathrm{G}}(\mathbf{C}) \times_{\mathbb{Q}_{\mathbf{C}}} \overline{\mathbb{Q}}$. Indeed, the fact that this subvariety is defined over $\mathbb{Q}_{\mathbf{C}}$ results from (i) $H_{\mathrm{G}}(\mathbf{C})$ is defined over $\mathbb{Q}_{\mathbf{C}}$, (ii) for any $\mathbf{p} \in H_{\mathrm{G}}(\mathbf{C})(\overline{\mathbb{Q}})$ corresponding to the G-isomorphism class of a G-cover $f,{ }^{\sigma} \mathbf{p}$ corresponds to the G-isomorphism class of the G-cover ${ }^{\sigma} f$, $\sigma \in \Gamma_{\mathbb{Q}_{\mathbf{C}}}$ and (iii) for any G-cover $f$ over $\overline{\mathbb{Q}}, \mathcal{E}_{\sigma f}=\mathcal{E}_{f}, \sigma \in \Gamma_{\mathbb{Q}_{\mathbf{C}}}$. Properties (ii) and (iii) imply that ${ }^{\sigma} H_{\mathrm{G}}(\mathbf{C}, \supset \mathcal{E})=H_{\mathrm{G}}(\mathbf{C}, \supset \mathcal{E}), \sigma \in \Gamma_{\mathbb{Q}_{\mathbf{C}}}$ and property (i) allows us to apply the Galois criterion for rationality [2, AG, Th. 14.4].

Corollary 1.3. For any conjugacy class $\mathcal{E}$ of finite subgroups of $\mathrm{PGL}_{2}(\overline{\mathbb{Q}})$, the set $H_{\mathrm{G} / \mathrm{PGL}_{2}}(\mathbf{C}, \supset \mathcal{E})$ is closed in $H_{\mathrm{G} / \mathrm{PGL}_{2}}(\mathbf{C})$. In particular, it can be equipped with a unique structure of reduced closed subvariety of $H_{\mathrm{G} / \mathrm{PGL}_{2}}(\mathbf{C})$ defined over $\mathbb{Q}$.

Proof. As the quotient morphism $\pi: H_{\mathrm{G}}(\mathbf{C}) \rightarrow H_{\mathrm{G} / \mathrm{PGL}_{2}}(\mathbf{C})$ is submersive and $H_{\mathrm{G}}(\mathbf{C}, \supset \mathcal{E})=\pi^{-1}\left(H_{\mathrm{G} / \mathrm{PGL}_{2}}(\mathbf{C}, \supset \mathcal{E})\right)$, theorem 1.2 also implies that the set $H_{\mathrm{G} / \mathrm{PGL}_{2}}(\mathbf{C}, \supset \mathcal{E})$ is closed in $H_{\mathrm{G} / \mathrm{PGL}_{2}}(\mathbf{C})$ and hence, by the same argument as for theorem 1.2, $H_{\mathrm{G} / \mathrm{PGL}_{2}}(\mathbf{C}, \supset \mathcal{E})$ can be equipped with a unique structure of reduced closed subvariety of $H_{\mathrm{G} / \mathrm{PGL}_{2}}(\mathbf{C})$ defined over $\mathbb{Q}_{\mathbf{C}}$.

Theorem 1.2 thus provides a stratification of Hurwitz spaces by closed subvarieties which encodes arithmetical properties of the morphism $\pi$.

We present two approaches to theorem 1.2. The first approach (section 3) relies on results from Mumford's geometric invariant theory and uses that $H_{\mathrm{G} / \mathrm{PGL}_{2}}(\mathbf{C})$ is the universal geometric quotient of $H_{\mathrm{G}}(\mathbf{C})$ under $\mathrm{PGL}_{2}$. The second approach (section 4) exploits the functorial properties of the Hurwitz schemes $H_{\mathrm{G} / \mathrm{PGL}_{2}}(\mathbf{C})$ constructed in [1]. This second approach is more explicit and, in particular, it provides a natural method to describe the geometrically irreducible components of our strata (section 4.2.3) and to search for $\mathbb{Q}$-rational points on $H_{G}(\mathbf{C})$ (section $5)$. 
Remark 1.4. Theorem 1.2 and corollary 1.3 could be extended to base fields $k$ of positive characteristic $p>0$ provided $p$ divides neither $|G|$ nor the (common) order $|E|$ of $E \in \mathcal{E}$ (take for instance $p \nmid|G| r$ !): under these assumptions, our proofs work without any change. However, for primes $p>0$ which do not divide $|G|$ but may divide $|E|$, our proofs would not work, at least as they are. More specifically, a key theorem 3.3 of our first approach fails in general. (If $A:=\mathrm{PGL}_{2}$ and $a:=\left(\begin{array}{ll}1 & 1 \\ 0 & 1\end{array}\right)$, we have $C(a)=A_{p} \backslash\left\{1_{A}\right\}\left(A_{p}:=\left\{a \in A \mid a^{p}=1_{A}\right\}\right)$, which is not closed in A.) Also, our second approach no longer works as it is since it resorts to coarse moduli schemes associated with groups of order $|G||E|$. Anyway, to avoid technical details, we only deal with the case of characteristic 0 .

\section{A DESCRIPTION OF $H_{g(\mathbf{C}), G, \mathbf{C}}$}

Let $S$ be a scheme and $\mathcal{X} \rightarrow(\mathrm{Sch} / S)$ a category fibered in groupoids over the category ( $\mathrm{Sch} / S$ ) of $S$-schemes (which we will shorten by "an $S$-groupoid"). Any $S$-scheme $X \rightarrow S$ is naturally identified with an $S$-groupoid by considering $(\mathrm{Sch} / X) \rightarrow(\mathrm{Sch} / S)$. A coarse moduli scheme for $\mathcal{X} \rightarrow(\mathrm{Sch} / S)$ is an $S$-scheme $X \rightarrow S$ together with an $S$-groupoid morphism $F: \mathcal{X} \rightarrow X$ such that (i) for any geometric point $k \rightarrow S, F(k): \mathcal{X}(k) \rightarrow X(k)$ induces a bijection between the isomorphism classes of objects in $\mathcal{X}(k)$ and $X(k)$ (which we will shorten by "is a bijection") and (ii) for any $S$-scheme $X^{\prime} \rightarrow S$ and any $S$-groupoid morphism $F^{\prime}: \mathcal{X} \rightarrow X^{\prime}$, there exists a unique morphism $\phi: X \rightarrow X^{\prime}$ in $\mathcal{C}$ such that $\phi \circ F=F^{\prime}$.

Note that a coarse moduli scheme - when it exists - is unique (by condition (ii)) and, also, that from the definition of an $S$-groupoid morphism, for any $k$-rational point $k \rightarrow S$ ( $k$ : a field), the bijection $F(\bar{k}): \mathcal{X}(\bar{k}) \rightarrow X(\bar{k})$ is compatible with the natural action of $\Gamma_{k}$, that is, for any $x \in \mathcal{X}(\bar{k}), \sigma \in \Gamma_{k}$, one has $F(\bar{k})\left(x^{\sigma}\right)=F(\bar{k})(x)^{\sigma}$.

2.1. Notation for Hurwitz spaces. Fix a finite group $G$ and an element $\mathbf{C} \in$ $\mathrm{R}_{+}^{*}(G)$. Set $\mathbb{Q}_{\mathbf{C}}:=\overline{\mathbb{Q}}^{\Delta_{\mathbf{C}}}$, where $\Delta_{\mathbf{C}} \subset \Gamma_{\mathbb{Q}}$ is the closed subgroup of all $s \in \Gamma_{\mathbb{Q}}$ such that $\mathbf{C}^{\chi(s)}=\mathbf{C}$, where $\chi: \Gamma_{k} \rightarrow \widehat{\mathbb{Z}}^{\times}$is the cyclotomic character of $k$. Finally, let $\mathbb{Z}_{\mathbf{C}}$ denote the ring of integers of $\mathbb{Q}_{\mathbf{C}}$.

Given a conjugacy class $C$ of $G$ we denote by $o(C)$ the common order of the 
elements in $C$. With this notation, we associate to $\mathbf{C}$ the corresponding genus $g(\mathbf{C})$ defined by the Riemann-Hurwitz formula

$$
g(\mathbf{C})=\frac{|G|}{2}\left(-2+\sum_{C \in \mathcal{C}(G)} n(C)\left(1-\frac{1}{o(C)}\right)\right)+1 .
$$

We retain the notation $\mathcal{H}_{\mathrm{G}}(\mathbf{C}), \mathcal{H}_{\mathrm{G} / \mathrm{PGL}_{2}}(\mathbf{C})$ and $\mathcal{H}_{g, G, \mathbf{C}}$ of section 1. Also, for an integer $r \geq 0$ (resp. integers $g, r \geq 0$ with $2-2 g-r<0$ ), we denote by $\mathcal{U}_{r}$ (resp. $\mathcal{M}_{g,[r]}$ ) the $\mathbb{Z}$-stack of projective lines (resp. genus $g$ curves) with $r$ unordered marked points (or, more precisely, relatively étale divisors of degree $r$ ) and by $U_{r}$ (resp. $M_{g,[r]}$ ) its coarse moduli scheme. (Thus, $\mathcal{U}_{r}=U_{r}$, i.e., $\mathcal{U}_{r}$ is representable.) Set $r=\operatorname{deg}(\mathbf{C})$. Then there are natural prestack morphisms $\mathcal{H}_{\mathrm{G}}(\mathbf{C}) \rightarrow \mathcal{U}_{r}, \mathcal{H}_{\mathrm{G} / \mathrm{PGL}_{2}}(\mathbf{C}) \rightarrow \mathcal{U}_{r} / \mathrm{PGL}_{2}, \mathcal{H}_{g, G, \mathbf{C}} \rightarrow \mathcal{M}_{g,[a]}$ and $\mathcal{H}_{g, G, \mathbf{C}} \rightarrow \mathcal{M}_{g^{\prime},[r]}$, where

$$
\begin{gathered}
a:=|G| \sum_{C \in \mathcal{C}(G)} \frac{n(C)}{o(C)}=|G|\left(2 g^{\prime}-2+r\right)-(2 g-2), \\
g^{\prime}:=1+\frac{g-1}{|G|}-\frac{1}{2} \sum_{C \in \mathcal{C}(G)} n(C)\left(1-\frac{1}{o(C)}\right) .
\end{gathered}
$$

(If $g^{\prime}$ is not an integer $\geq 0$, set $\mathcal{M}_{g^{\prime},[r]}=\emptyset$.)

From [16], the $\mathbb{Z}_{\mathbf{C}}\left[\frac{1}{G]}\right]$-stack $\mathcal{H}_{\mathrm{G}}(\mathbf{C})$ is an algebraic stack proper, quasi-finite and étale over $\mathcal{U}_{r}$; it admits a coarse moduli scheme $\mathcal{H}_{\mathrm{G}}(\mathbf{C}) \rightarrow H_{\mathrm{G}}(\mathbf{C})$, which is an affine, smooth $\mathbb{Z}_{\mathbf{C}}\left[\frac{1}{|G|}\right]$-scheme finite étale over $U_{r}$.

From [1, Th. 6.9 and Prop. 6.14], the $\mathbb{Z}_{\mathbf{C}}\left[\frac{1}{|G|}\right]$-stack $\mathcal{H}_{g, G, \mathbf{C}}$ admits a stable compactification $\overline{\mathcal{H}}_{g, G, \mathbf{C}}$, which is a Deligne-Mumford stack smooth and proper over $\mathbb{Z}_{\mathbf{C}}\left[\frac{1}{\mid G]}\right]$. The natural extended morphism $\overline{\mathcal{H}}_{g, G, \mathbf{C}} \rightarrow \overline{\mathcal{M}}_{g,[a]}$ is finite (representable) and unramified whereas the natural extended morphism $\overline{\mathcal{H}}_{g, G, \mathbf{C}} \rightarrow$ $\overline{\mathcal{M}}_{g^{\prime},[r]}$ is proper, quasi-finite and flat (but representable if and only if either $G$ has trivial center or $\left.\overline{\mathcal{H}}_{g, G, \mathbf{C}}=\emptyset\right)$; at the level of coarse moduli spaces, the latter is finite and surjective. We will only consider the coarse moduli scheme $\mathcal{H}_{g, G, \mathbf{C}} \rightarrow H_{g, G, \mathbf{C}}$, which is normal, of finite type over $\mathbb{Z}_{\mathbf{C}}\left[\frac{1}{|G|}\right]$ and quasi-finite over $M_{g,[a]} \cdot{ }^{3}$

\footnotetext{
$3 \overline{\text { In fact, } H_{g, G, \mathbf{C}}}$ is finite over $M_{g,[a]}$, which is crucial in our second approach. See the proof of lemma 4.3 .
} 
2.2. A description of $H_{g(\mathbf{C}), G, \mathbf{C}}$. Recall that $S \mapsto \mathcal{H}_{\mathrm{G} / \mathrm{PGL}_{2}}(\mathbf{C})(S)$ is a prestack over $\mathbb{Z}_{\mathbf{C}}\left[\frac{1}{[G]}\right]$ but not a stack in general; its associated stack [8, Chapter 3] is

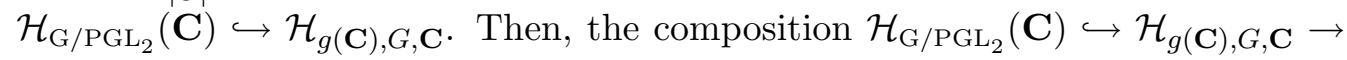
$H_{g(\mathbf{C}), G, \mathbf{C}}$ is a coarse moduli scheme for $\mathcal{H}_{\mathrm{G} / \mathrm{PGL}_{2}}(\mathbf{C})$; condition (i) is straightforward and condition (ii) results from the universal property of the stack associated to a prestack.

Let $\mathcal{H}_{G}(\mathbf{C})^{*}$ denote the presheaf of isomorphism classes $S \mapsto \mathcal{H}_{G}(\mathbf{C}) / \simeq$. Then the $\mathbb{Z}_{\mathbf{C}}\left[\frac{1}{|G|}\right]$-group scheme $\mathrm{PGL}_{2}$ acts naturally on $\mathcal{H}_{G}(\mathbf{C})^{*}$ (by translation on the left). By condition (ii) of the definition of coarse moduli scheme, this action induces an algebraic action defined over $\mathbb{Z}_{\mathbf{C}}\left[\frac{1}{|G|}\right]$ of $\mathrm{PGL}_{2}$ on $H_{\mathrm{G}}(\mathbf{C})$. We classically denote the corresponding quotient stack by $\left[H_{\mathrm{G}}(\mathbf{C}) / \mathrm{PGL}_{2} / \mathbb{Z}_{\mathbf{C}}\left[\frac{1}{|G|}\right]\right][8$, Ex. 2.4.2].

We explain below that the $\mathbb{Q}_{\mathbf{C}}$-stack $\left[H_{\mathrm{G}}(\mathbf{C}) / \mathrm{PGL}_{2} / \mathbb{Q}_{\mathbf{C}}\right]$ admits a coarse moduli scheme.

Let $k$ be a field of characteristic 0 and let $A$ be a connected reductive $k$-group acting on an affine $k$-scheme of finite type $X$ via $\sigma: A \times_{k} X \rightarrow X$. Consider the following cartesian square

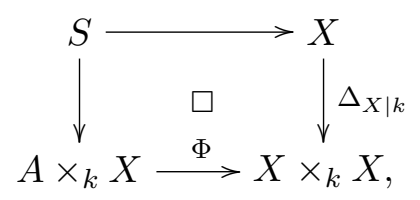

where $\Phi$ is the map $A \times_{k} X \rightarrow X \times_{k} X$ sending $(a, x)$ to $(a x, x)$. Then $S \rightarrow X$ is a closed subgroup scheme of $A \times_{k} X \stackrel{p_{2}}{\rightarrow} X$ called the stabilizer group scheme. Also, given a ( $k$-rational) geometric point $x \in X$, the orbit of $x$ is the subset $O(x)=\Phi\left(A \times_{k} x\right) \subset X$; it is a smooth locally closed subvariety of $X[2$, I.1.8, Prop.].

A categorical quotient for $\sigma$ is a $k$-morphism $\pi: X \rightarrow X / A$ such that (i) $\pi \circ \sigma=\pi \circ p_{2}$ and (ii) for any $k$-morphism $\phi: X \rightarrow Y$ such that $\phi \circ \sigma=\phi \circ p_{2}$ holds there is a unique $k$-morphism $\bar{\phi}: X / A \rightarrow Y$ such that $\bar{\phi} \circ \pi=\phi$. A geometric quotient for $\sigma$ is a $k$-morphism $\pi: X \rightarrow X / A$ such that (i) $\pi \circ \sigma=\pi \circ p_{2}$, (ii) for any geometric point $x \in X$, the fiber $\pi^{-1}(\pi(x))$ is precisely $O(x)$, (iii) $\pi$ is submersive (i.e. $U \subset X / A$ is open if and only if $\pi^{-1}(U) \subset X$ is open in $X$.) and (iv) $\mathcal{O}_{X / A}=\left(\pi_{*} \mathcal{O}_{X}\right)^{A}$. A geometric quotient is always a categorical quotient [11, Prop. 0.1], and, in particular, is unique up to a unique isomorphism. A universal 
geometric quotient ${ }^{4}$ for $\sigma$ is - when it exists - a coarse moduli scheme for the $k$-stack $[X / A / k][8$, Chap. 19].

Lemma 2.1. Assume that $A$ acts on $X$ via $\sigma$ with finite stabilizers, then there exists a universal geometric quotient $\pi: X \rightarrow X / A$ for $\sigma$.

Proof. Lemma 2.1 is a consequence of geometric invariant theory. By [11, Th. 1.1], a universal categorical quotient $\pi: X \rightarrow X / A$ exists for $\sigma$ with $X / A$ affine and $\pi$ universally submersive. Furthermore, by [11, Amp. 1.3], this quotient is a (universal) geometric quotient if and only if $\sigma$ is closed, that is, for any geometric point $x \in X$ the orbit $O(x) \subset X$ is closed in $X$. So, lemma 2.1 amounts to proving that $\sigma$ is closed. For each $x \in X(k)$, the natural surjective morphism $A \rightarrow O(x), a \mapsto a x$ is quasi-finite by assumption. Thus, $\operatorname{dim}(O(x))=\operatorname{dim}(A)$ (see [7, II, ex. 3.22]) is independent of $x$. Now the assertion follows from the so-called closed orbit lemma [2, I.1.8, Prop.].

We deduce from lemma 2.1 that there exists a universal geometric quotient $\pi$ : $H_{\mathrm{G}}(\mathbf{C}) \rightarrow H_{\mathrm{G} / \mathrm{PGL}_{2}}^{\text {gen }}(\mathbf{C})$ defined over $\mathbb{Q}_{\mathbf{C}}$. The notation $H_{\mathrm{G} / \mathrm{PGL}_{2}}^{\text {gen }}(\mathbf{C})$ is explained by the following lemma.

Lemma 2.2. The $\mathbb{Q}_{\mathbf{C}}$-scheme $H_{\mathrm{G} / \mathrm{PGL}_{2}}^{\text {gen }}(\mathbf{C})$ is canonically isomorphic to the generic fiber of the $\mathbb{Z}_{\mathbf{C}}\left[\frac{1}{|G|}\right]$-scheme $H_{\mathrm{G} / \mathrm{PGL}_{2}}(\mathbf{C})$.

Proof. When $G$ has trivial center, this is straightforward since $\mathcal{H}_{\mathrm{G}}(\mathbf{C})=H_{\mathrm{G}}(\mathbf{C})$. Else, it formally results from condition (ii) of the definition of coarse moduli scheme. Indeed, consider the following commutative diagram, which sums up the situation and where the first row has to be considered as restricted to the category of $\mathbb{Q}_{\mathbf{C}}$-schemes.

$(2)$

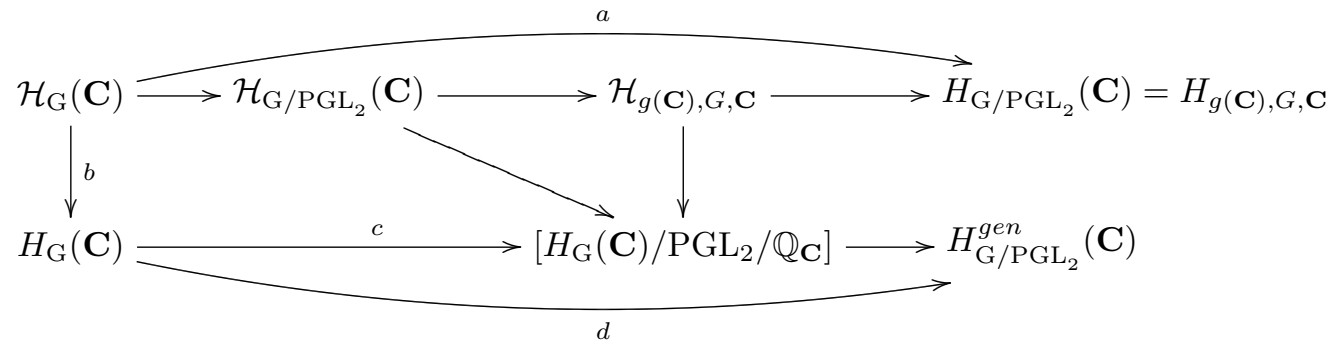

${ }^{4}$ For the definition of universal categorical/geometric quotients, see [11, Def. 0.7]. 


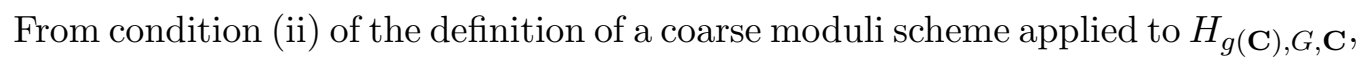
there exists a unique morphism $\alpha: H_{g(\mathbf{C}), G, \mathbf{C}} \rightarrow H_{\mathrm{G} / \mathrm{PGL}_{2}}^{g e n}(\mathbf{C})$ making the last square commute. Also, the same condition applied to $H_{\mathrm{G}}(\mathbf{C})$ yields a unique morphism $\beta: H_{\mathrm{G}}(\mathbf{C}) \rightarrow H_{g(\mathbf{C}), G, \mathbf{C}}$ such that $\beta \circ b=a$. This morphism, in turn, factors through $c$ and condition (ii) of the definition of a coarse moduli scheme applied to $H_{\mathrm{G} / \mathrm{PGL}_{2}}^{g e n}(\mathbf{C})$ yields a unique morphism $\gamma: H_{\mathrm{G} / \mathrm{PGL}_{2}}^{g e n}(\mathbf{C}) \rightarrow H_{g(\mathbf{C}), G, \mathbf{C}}$ such that $\gamma \circ d=\beta$. Then, invoking one more time condition (ii) of the definition of a coarse moduli scheme, one gets $\alpha \circ \gamma=\mathrm{Id}$ and $\gamma \circ \alpha=\mathrm{Id}$.

In the following, we will no longer distinguish between $H_{\mathrm{G} / \mathrm{PGL}_{2}}^{g e n}(\mathbf{C})$ and the generic fiber of $H_{\mathrm{G} / \mathrm{PGL}_{2}}(\mathbf{C})$, both of which we will denote by $H_{\mathrm{G} / \mathrm{PGL}_{2}}(\mathbf{C})$.

\section{First APPROACH}

We retain the notation of section 2.2 .

Lemma 3.1. Assume that $A$ acts on $X$ with finite stabilizers, then the map $\Phi: A \times_{k} X \rightarrow X \times_{k} X$ is proper and unramified.

Proof. Since $\sigma$ is closed (see the proof of Lemma 2.1) and $\pi$ is affine, the properness of $\Phi$ follows from [11, Prop. 0.8 ]. The unramifiedness of $\Phi$ follows from the fact that, for each geometric point $(x, y)$ of $X \times_{k} X$, the fiber of $\Phi$ at $(x, y)$ is either empty or an $S_{x}$-torsor, where $S_{x}$ is the fiber at $x$ of the stabilizer group scheme $S \rightarrow X$. (Note that any finite group scheme over a field of characteristic 0 is étale.)

Corollary 3.2. Fix $a_{1}, \ldots, a_{m} \in A$ and let $E$ be the subgroup of $A$ generated by $a_{1}, \ldots, a_{m}$. Assume that the $A$-orbit $C\left(a_{1}, \ldots, a_{m}\right)$ of $\left(a_{1}, \ldots, a_{m}\right)$ (with respect to the diagonal, conjugate action) is closed in $A^{m}$. Then the set of points $x \in X$ such that $S_{x}$ contains a conjugate of $E$ is closed in $X$.

Proof. By lemma 3.1 applied to $A^{m}$ acting on $X^{m}$ by the direct product action induced by $\sigma: A \times_{k} X \rightarrow X$, the set $\Phi\left(C\left(a_{1}, \ldots, a_{m}\right) \times_{k} X^{m}\right)$ is closed in $X^{m} \times_{k} X^{m}$. Let $\Delta_{X \mid k}^{m}: X \rightarrow X^{m}$ denote the $m$ th diagonal map over $k$ and set $\Delta_{X \mid k}^{m, 2}=\Delta_{X \mid k}^{m} \times_{k} \Delta_{X \mid k}^{m} \circ \Delta_{X \mid k}^{2}$. Then the set $\left(\Delta_{X \mid k}^{m, 2}\right)^{-1}\left(\Phi\left(C\left(a_{1}, \ldots, a_{m}\right) \times_{k} X^{m}\right)\right)$ 
is closed in $X$, which yields the announced conclusion.

Combined with the following result from the theory of algebraic groups, corollary 3.2 yields theorem 1.2.

Theorem 3.3. With the notation of corollary 3.2 and assuming furthermore that $E$ is finite, the $A$-orbit $C\left(a_{1}, \ldots, a_{m}\right)$ is closed in $A^{m}$.

Proof. This is a special case of [12, Th. 2.16].

However, it is possible to prove theorem 1.2 in most cases without resorting to theorem 3.3. This relies on a group-theoretic lemma and a semi-continuity argument combined with the elementary case of theorem 1.2 for cyclic groups proved in example 3.4. The rest of this section is devoted to this elementary (partial) proof of theorem 1.2.

Example 3.4. Consider $A:=\mathrm{PGL}_{2}$. Then, as $A$ is separated, the set $A_{n}:=\{a \in$ $\left.A \mid a^{n}=1_{A}\right\}$ is closed for any integer $n \geq 1$. Furthermore, there are exactly $\phi(n) / 2$ conjugacy classes of order $n$ elements in $\mathrm{PGL}_{2}(\bar{k})$, namely the ones of $a_{n}^{l}:=\left(\begin{array}{cc}\zeta_{n}^{l} & 0 \\ 0 & 1\end{array}\right), l \in(\mathbb{Z} / n)^{\times} / \pm 1 .{ }^{5}$ Considering for instance the commutative diagram

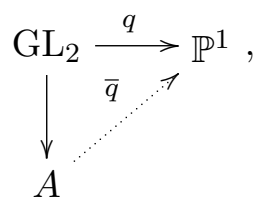

where $q(a)=\left[\operatorname{Tr}(a)^{2}-2 \operatorname{det}(a): \operatorname{det}(a)\right]$, one obtains that $C\left(a_{n}^{l}\right)=\bar{q}^{-1}\left(\left[\zeta_{n}^{l}+\zeta_{n}^{-l}\right.\right.$ : 1]) $\cap A_{n}$ is closed. Combined with corollary 3.2 (for $m=1$ ) this yields theorem 1.2 for cyclic groups.

Given a finite group $E$ denote by $I(E)_{n} \geq 0$ the number of elements with order exactly $n$ in $E$ and set $I(E)=\left(I(E)_{n}\right)_{n \geq 1}$. For any two sequences of integers $I_{i}=\left(I_{i, n}\right)_{n \geq 1}, i=1,2$ say that $I_{1} \leq I_{2}$ if $I_{1, n} \leq I_{2, n}, n \geq 1$. With these notations

${ }^{5}$ Indeed, if $a$ has order exactly $n$, then the cyclic subgroup $\langle a\rangle$ it generates is conjugate in $A$ to the group $C_{n}$ of lemma 1.1, hence $a$ is conjugate in $A$ to an element of the form $a_{n}^{l}$ for some $l \in(\mathbb{Z} / n)^{\times} / \pm 1$. Eventually, two elements $a_{n}^{l}$ and $a_{n}^{l^{\prime}}$ with $l \neq l^{\prime} \in(\mathbb{Z} / n)^{\times} / \pm 1$ are not conjugate in $A$. 
we have:

Lemma 3.5. Assume that $E_{1}, E_{2}$ are two groups isomorphic to one of the following:

- Cyclic group: $\quad C_{n}, n \geq 1$;

- Dihedral group: $\quad D_{2 n}=C_{n} \rtimes C_{2}, n \geq 2$;

- Symmetric group: $\mathcal{S}_{4}$;

- Alternating group: $\mathcal{A}_{4}, \mathcal{A}_{5}$.

If $I\left(E_{1}\right) \leq I\left(E_{2}\right)$ then one of the following holds:

(i) there exists a monomorphism $E_{1} \hookrightarrow E_{2}$;

(ii) $E_{1} \simeq D_{4}$ and $E_{2} \simeq D_{2 n}$ with $n \geq 3$ odd; or

(iii) $E_{1} \simeq D_{6}$ and $E_{2} \simeq A_{4}$.

Proof. Compute first the different possible values of $I(E)$ :

$$
\begin{array}{ll}
I\left(C_{n}\right)=\left(I\left(C_{n}\right)_{m}\right)_{m \geq 1} \text { with } \begin{array}{ll}
I\left(C_{n}\right)_{m}=I\left(C_{m}\right)_{m} & \text { if } m \mid n ; \\
& I\left(C_{n}\right)_{m}=0
\end{array} & \text { else; } \\
I\left(D_{2 n}\right)=\left(1, n+I\left(C_{n}\right)_{2},\left(I\left(C_{n}\right)_{m}\right)_{m \geq 3}\right) ; & \\
I\left(\mathcal{A}_{4}\right)=(1,3,8,0,0,0, \ldots) ; & \\
I\left(\mathcal{S}_{4}\right)=(1,9,8,6,0,0, \ldots) ; & \\
I\left(\mathcal{A}_{5}\right)=(1,15,20,0,24,0, \ldots) . &
\end{array}
$$

Also observe that $I\left(C_{n}\right)_{2} \leq 1, n \geq 2$ and $I\left(D_{2 n}\right)_{3} \leq 2, n \geq 2$. We now fix $E_{1}$ and consider what the condition $I\left(E_{1}\right) \leq I\left(E_{2}\right)$ imposes on $E_{2}$.

- If $I\left(C_{n}\right) \leq I(E)$ then, in particular, $1 \leq I\left(C_{n}\right)_{n} \leq I(E)_{n}$ that is $E$ contains at least one element of order $n$ thus $C_{n} \hookrightarrow E$.

- If $I\left(D_{2 n}\right) \leq I(E)$ then, in particular, $2 \leq I\left(D_{2 n}\right)_{2}=n+I\left(C_{n}\right)_{2} \leq I(E)_{2}$ thus $E$ is not cyclic and $1 \leq I\left(D_{2 n}\right)_{n}=n \leq I(E)_{n}$ thus $E$ contains at least one element of order $n$. So:

- - either $E$ is a dihedral group $D_{2 N}$ with $n \mid N$ thus $D_{2 n} \hookrightarrow E$;

- - or $E \simeq \mathcal{A}_{4}$ and $n+I\left(C_{n}\right)_{2} \leq I(E)_{2}=3$ so $n=2,3$. If $n=2$ we are in the situation $D_{4} \hookrightarrow \mathcal{A}_{4}$, if $n=3$ we are in the exceptional case $E_{1} \simeq D_{6}$ and $E_{2} \simeq \mathcal{A}_{4}$

- - or $E \simeq \mathcal{S}_{4}$ and $n+I\left(C_{n}\right)_{2} \leq I(E)_{2}=9$ so $2 \leq n \leq 9$. If $n=2,3,4$ we are in the case $D_{2 n} \hookrightarrow \mathcal{S}_{4}$ and the case $n \geq 5$ can never occur because $I\left(\mathcal{S}_{4}\right)_{n}=0$, $n \geq 5$;

- or $E \simeq \mathcal{A}_{5}$ and $n+I\left(C_{n}\right)_{2} \leq I(E)_{2}=15$ so $2 \leq n \leq 15$. If $n=2,3,5$ we 
are in the case $D_{2 n} \hookrightarrow \mathcal{A}_{5}$, the case $n=4$ can never occur because $I(E)_{4}=0$ and neither can the case $n \geq 6$ because $I\left(\mathcal{A}_{5}\right)_{n}=0, n \geq 6$.

- If $I\left(\mathcal{A}_{4}\right) \leq I(E)$ then the only possibilities for $E$ are $E \simeq \mathcal{A}_{4}, \mathcal{S}_{4}, \mathcal{A}_{5}$ and, in any case, $\mathcal{A}_{4} \hookrightarrow E$.

- If $I\left(\mathcal{S}_{4}\right) \leq I(E)$ then $E \simeq \mathcal{S}_{4}$.

- If $I\left(\mathcal{A}_{5}\right) \leq I(E)$ then $E \simeq \mathcal{A}_{5}$.

Corollary 3.6. Theorem 1.2 holds except possibly for the dihedral groups $D_{4}$ and $D_{6}$.

Proof. From now on $X / k$ stands for the coarse moduli scheme $H_{\mathrm{G}}(\mathbf{C}) \times{ }_{\mathbb{Q}_{\mathbf{C}}} \overline{\mathbb{Q}} / \overline{\mathbb{Q}}$ and $A$ stands for $\mathrm{PGL}_{2}$. For any $n \geq 1$ set $A_{n}^{0}:=\{a \in A||<a>\mid=n\}$. By example 3.4 this is a closed subset of $A$. (Indeed, $A_{n}^{0}$ is a (disjoint) union of $C\left(a_{n}^{l}\right)$ for $l \in(\mathbb{Z} / n)^{\times} / \pm 1$.) As a result, the morphism

$$
\Psi_{n}: A_{n}^{0} \times_{k} X \hookrightarrow A \times_{k} X \stackrel{\Phi}{\longrightarrow} X \times_{k} X
$$

is a finite morphism by lemma 3.1. In particular, by [7, II, ex. 5.8] the map

$$
\begin{aligned}
I_{n}: X \times_{k} X & \longrightarrow \mathbb{Z}_{\geq 0} \\
\omega & \mapsto \operatorname{dim}_{k(\omega)} \mathcal{O}_{A_{n}^{0} \times{ }_{k} X, \omega} \otimes_{\mathcal{O}_{X \times k} X, \omega} k(\omega)
\end{aligned}
$$

is upper semi-continuous. Observe that, for each geometric point $\omega$ of $X \times_{k} X$, we have $I_{n}(\omega)=\left|\Psi_{n}^{-1}(\omega)\right|$, since $\Psi_{n}$ is unramified by lemma 3.1. So, given any sequence of positive integers $I=\left(I_{n}\right)_{n \geq 1}$ the set

$$
\mathcal{L}(I):=\bigcap_{n \geq 1}\left\{\omega \in X \times_{k} X \mid I_{n}(\omega) \geq I_{n}\right\} \subset X \times_{k} X
$$

is closed thus so is $\Delta_{X \mid k}^{-1}(\mathcal{L}(I)) \subset X$. But $\Delta_{X \mid k}^{-1}(\mathcal{L}(I))=\left\{x \in X \mid I\left(S_{x}\right) \geq I\right\}$. Corollary 3.6 now results from lemma 3.5 and lemma 1.1.

Remark 3.7. The above arguments also work with $X \rightarrow k$ standing for $U_{r} \times_{\mathbb{Q}} \overline{\mathbb{Q}}$. In particular, if we denote by $U_{r}(\supset \mathcal{E})$ the subset of all points of $U_{r}$ corresponding to $r$-tuples $\mathbf{t}$ whose stabilizer contains a representative of $\mathcal{E}$, then we have also proved: Let $\mathcal{E}$ be a conjugacy class of finite subgroups in $\mathrm{PGL}_{2}(\overline{\mathbb{Q}})$ then the set $U_{r}(\supset \mathcal{E})$ is closed in $U_{r}$. In particular, it can be equipped with a unique structure of reduced closed subvariety of $U_{r}$ defined over $\mathbb{Q}$. 


\section{SeCOND APPROACH}

4.1. Preliminary remarks. The second approach interprets the base locus $H_{\mathrm{G}}(\mathbf{C}, \supset \mathcal{E})$ as the last element of a series of inverse and direct images of morphisms between moduli schemes. In the following, given an extension of finite groups $1 \rightarrow K \rightarrow F \stackrel{p}{\rightarrow} Q \rightarrow 1$, we will say that $Q$ acts trivially on $K$ if there exists a set-theoretic section $s: Q \rightarrow F$ of $p$ such that $s(q) k s(q)^{-1}=k, q \in Q, k \in K$. This is equivalent to requiring that the canonical representation $Q \rightarrow \operatorname{Out}(K)$ be trivial. To explain our second approach, start with a G-cover $f: X \rightarrow \mathbb{P}^{1}$ with group $G$ and with a nontrivial base group $E$ and consider the resulting cover $\tilde{f}: X \stackrel{f}{\rightarrow} \mathbb{P}^{1} \stackrel{\Pi}{\rightarrow} \mathbb{P}^{1} / E$. Fixing an isomorphism $i: \mathbb{P}^{1} / E \simeq \mathbb{P}^{1}$ we obtain a cover $i \circ \tilde{f}: X \rightarrow \mathbb{P}^{1}$ which, by definition of the base group, is a G-cover with group, say, $\tilde{E}$. We can make the following three remarks:

(1) The group $\tilde{E}$ is an extension $1 \rightarrow G \rightarrow \tilde{E} \stackrel{p}{\rightarrow} E \rightarrow 1$ where $E$ acts trivially on $G$. Indeed, by definition of the base group, any $e \in E$ admits a lift $\epsilon$ to $\operatorname{Aut}(X)$ which is compatible with $\operatorname{Aut}(f) \stackrel{\alpha}{\rightarrow} G$ that is, $\alpha\left(\epsilon g \epsilon^{-1}\right)=\alpha(g)$, $g \in \operatorname{Aut}(f)$ and, hence, $\epsilon g \epsilon^{-1}=g, g \in G$. In the following, we will denote by $\mathcal{E}(E, G)$ the set of all group extensions $1 \rightarrow G \rightarrow \tilde{E} \stackrel{p}{\rightarrow} E \rightarrow 1$ with $E$ acting trivially on $G$. This set is always non-empty since it contains the direct product $\tilde{E}=G \times E$. Hence, by classical group cohomology, there is a canonical bijection $(\mathcal{E}(E, G) / \simeq) \tilde{\rightarrow} \mathrm{H}^{2}(E, Z(G))$ sending the direct product $G \times E$ to the trivial class in $\mathrm{H}^{2}(E, Z(G))$. In particular, when $Z(G)$ is trivial, the only possible extension is $\tilde{E}=G \times E$.

(2) The cover $i \circ \Pi: \mathbb{P}^{1} \rightarrow \mathbb{P}^{1}$ is ramified over two or three points. Indeed, first, recall that there is no nontrivial cover of $\mathbb{P}^{1}$ with 0 or 1 ramification point (and by assumption, $E$ is nontrivial). Also, $i \circ \Pi$ is a G-cover with group $E$. Thus, if $e_{1}, \ldots, e_{s}$ denote the list of the ramification indices $>1$, the Riemann-Hurwitz formula becomes

$$
0>-2=|E|\left(-2+\sum_{1 \leq i \leq s}\left(1-\frac{1}{e_{i}}\right)\right) \geq|E|\left(-2+\frac{s}{2}\right)
$$

This implies $s<4$. 
(3) If we assume furthermore that $f: X \rightarrow \mathbb{P}^{1}$ is ramified over $r \geq 3$ points then $X$ has genus $\geq 1$. Else, $\tilde{E}$ would necessarily be one of the groups listed in lemma 1.1. In particular, the extension $1 \rightarrow G \rightarrow \tilde{E} \stackrel{p}{\rightarrow} E \rightarrow 1$ would be one of the following

$$
\begin{aligned}
& 1 \rightarrow C_{n} \rightarrow C_{n m} \stackrel{p}{\rightarrow} C_{m} \rightarrow 1, n, m \geq 2 ; \\
& 1 \rightarrow C_{n} \rightarrow D_{2 n m} \stackrel{p}{\rightarrow} D_{2 m} \rightarrow 1, n, m \geq 2 \text {; } \\
& 1 \rightarrow C_{n} \rightarrow D_{2 n} \stackrel{p}{\rightarrow} C_{2} \rightarrow 1, n \geq 2 ; \\
& 1 \rightarrow D_{4} \rightarrow \mathcal{A}_{4} \stackrel{p}{\rightarrow} C_{3} \rightarrow 1 ; \\
& 1 \rightarrow D_{4} \rightarrow \mathcal{S}_{4} \stackrel{p}{\rightarrow} D_{6} \rightarrow 1 \text {; } \\
& 1 \rightarrow \mathcal{A}_{4} \rightarrow \mathcal{S}_{4} \stackrel{p}{\rightarrow} C_{2} \rightarrow 1 .
\end{aligned}
$$

Then, recalling that $E$ acts trivially on $G$, the only remaining cases are $1 \rightarrow$ $C_{2} \rightarrow D_{4 m} \rightarrow D_{2 m} \rightarrow 1, m \geq 2,1 \rightarrow C_{n} \rightarrow C_{n m} \stackrel{p}{\rightarrow} C_{m} \rightarrow 1, n, m \geq 2$ and $1 \rightarrow C_{2} \rightarrow D_{4} \stackrel{p}{\rightarrow} C_{2} \rightarrow 1$. In particular, they all correspond to a cyclic group $G$. But, by the Riemann-Hurwitz formula, a nontrivial cyclic G-cover $f: \mathbb{P}^{1} \rightarrow \mathbb{P}^{1}$ ramifies above exactly two points, which contradicts $r \geq 3$.

Also, $i \circ \tilde{f}$ is only defined after fixing an isomorphism $i: \mathbb{P}^{1} / E \simeq \mathbb{P}^{1}$ but, for two such choices $i_{1}, i_{2}$, the resulting covers $i_{1} \circ \tilde{f}, i_{2} \circ \tilde{f}$ are G/PGL $\mathrm{PL}_{2}$ isomorphic and, thus, the $\mathrm{G} / \mathrm{PGL}_{2}$-isomorphism class of $i \circ \tilde{f}$ no longer depends on $i$; we denote it by $\tilde{f}$. Similarly, we denote by $\Pi$ the $\mathrm{G} / \mathrm{PGL}_{2}$-isomorphism class of $i \circ \Pi$. The idea is to reverse this construction and recover $f$ from $\Pi$ and $\tilde{f}$.

\subsection{Second proof of theorem $\mathbf{1 . 2}$.}

4.2.1. Notation. First, we introduce some notations. We write $\mathrm{R}_{+}(G) \rightarrow \mathrm{R}_{+}^{*}(G)$, $\mathbf{C}=\sum_{C \in \mathcal{C}} n(C) C \mapsto \mathbf{C}^{*}=\sum_{C \in \mathcal{C}^{*}} n(C) C$ for the canonical projection. Observe that $g\left(\mathbf{C}^{*}\right)=g(\mathbf{C})$ and $\operatorname{deg}\left(\mathbf{C}^{*}\right)=\operatorname{deg}(\mathbf{C})-n(\{1\})$ hold.

Then any group epimorphism $p: \tilde{E} \rightarrow E$ defines a morphism of monoids $\mu: \mathrm{R}_{+}(\tilde{E}) \rightarrow \mathrm{R}_{+}(E)$, sending $\tilde{C} \in \mathcal{C}(\tilde{E})$ to $p(\tilde{C}) \in \mathcal{C}(E)$. Geometrically, if $\tilde{f}: X \rightarrow \mathbb{P}^{1}$ is a G-cover with group $\tilde{E}$ and inertia canonical invariant $\tilde{\mathbf{C}}$ then $\mu^{*}(\tilde{\mathbf{C}}):=\mu(\tilde{\mathbf{C}})^{*}$ is the inertia canonical invariant of the G-cover $X / G \rightarrow \mathbb{P}^{1}$ with group $E$.

Similarly, any group monomorphism $i: G \hookrightarrow \tilde{E}$ defines a morphism of monoids $\nu: \mathrm{R}_{+}(\tilde{E}) \rightarrow \mathrm{R}_{+}(G)$ as follows. Consider the canonical map $C_{\mathrm{G}}: G \rightarrow \mathcal{C}(G)$, 
sending $g \in G$ to its conjugacy class $C_{G}(g)$ in $G$ and let $s: \mathcal{C}(G) \hookrightarrow G, C \rightarrow s(C)$ a section of it. Then $\nu$ sends $\tilde{C} \in \mathcal{C}(\tilde{E})$ to

$$
\sum_{\tilde{e} \in G \backslash \tilde{E} /<s(\tilde{C})>} C_{G}\left(\tilde{e} s(\tilde{C})^{\frac{o(\tilde{C})}{\left|G \cap \tilde{e}<s(\tilde{C})>\tilde{e}^{-1}\right|}} \tilde{e}^{-1}\right) \in \mathrm{R}_{+}(G) .
$$

Geometrically, if $\tilde{f}: X \rightarrow \mathbb{P}^{1}$ is a G-cover with group $\tilde{E}$ and inertia canonical invariant $\tilde{\mathbf{C}}$ then $\nu^{*}(\tilde{\mathbf{C}}):=\nu(\tilde{\mathbf{C}})^{*}$ is the inertia canonical invariant of the G-cover $X \rightarrow X / G$ with group $G$. Note that for an extension of finite groups $1 \rightarrow G \stackrel{i}{\rightarrow} \tilde{E} \stackrel{p}{\rightarrow} E \rightarrow 1$ where $E$ acts trivially on $G$ and for any $\tilde{C} \in \mathcal{C}(\tilde{E})$, the set $\left\{g^{o(p(\tilde{C}))} \mid g \in \tilde{C}\right\}$ generates exactly one conjugacy class in $G$; we denote it by $\tilde{C}^{o(p(\tilde{C}))}$. Then $\nu(\tilde{C})$ is simply $\frac{|E|}{o(p(\tilde{C}))} \tilde{C}^{o(p(\tilde{C}))} \in \mathrm{R}_{+}(G)$.

4.2.2. Second proof of theorem 1.2. From now on, let $1 \rightarrow G \stackrel{i}{\rightarrow} \tilde{E} \stackrel{p}{\rightarrow} E \rightarrow 1$ be an extension of finite groups where $E$ acts trivially on $G$.

Lemma 4.1. For each $\mathbf{C} \in \mathrm{R}_{+}^{*}(G)$ the set of all $\tilde{\mathbf{C}} \in\left(\nu^{*}\right)^{-1}(\mathbf{C}) \cap \mathrm{R}_{+}^{*}(\tilde{E})$ such that $g\left(\mu^{*}(\tilde{\mathbf{C}})\right)=0$ is finite.

Proof. Fix $\mathbf{C} \in \mathrm{R}_{+}^{*}(G)$ and $\tilde{\mathbf{C}} \in\left(\nu^{*}\right)^{-1}(\mathbf{C}) \cap \mathrm{R}_{+}^{*}(\tilde{E})$ such that $g\left(\mu^{*}(\tilde{\mathbf{C}})\right)=0$. Then we have

$$
\operatorname{deg}(\tilde{\mathbf{C}}) \leq \frac{\operatorname{deg}(\mathbf{C})}{|E|}+\operatorname{deg}\left(\mu^{*}(\tilde{\mathbf{C}})\right) \leq \frac{\operatorname{deg}(\mathbf{C})}{|E|}+3,
$$

where the last inequality is implied by $g\left(\mu^{*}(\tilde{\mathbf{C}})\right)=0$.

Now, fix an integer $r \geq 3$, a finite nontrivial subgroup $E$ of $\mathrm{PGL}_{2}(\overline{\mathbb{Q}})$ and a degree $r$ element $\mathbf{C} \in \mathrm{R}_{+}^{*}(G)$. Define

$$
H_{\mathrm{G} / \mathrm{PGL}_{2}}^{0}(\mathbf{C}, E):=\coprod_{(\tilde{E}, p) \in \mathcal{E}(E, G) / \simeq \tilde{\mathbf{C} \in\left(\nu^{*}\right)^{-1}(\mathbf{C}) \cap \mathrm{R}_{+}^{*}(\tilde{E})}} H_{\left.\mathrm{G}^{\prime} / \mu^{*}(\tilde{\mathbf{C}})\right)=0}(\tilde{\mathbf{C}})
$$

Note that, by point (3) above, the defining conditions $r \geq 3, \tilde{\mathbf{C}} \in\left(\nu^{*}\right)^{-1}(\mathbf{C}) \cap$ $\mathrm{R}_{+}^{*}(\tilde{E})$ and $g\left(\mu^{*}(\tilde{\mathbf{C}})\right)=0$ force $g(\mathbf{C})=g(\tilde{\mathbf{C}}) \geq 1$ (hence, in particular, $\operatorname{deg}(\tilde{\mathbf{C}}) \geq$ 3). Accordingly, $H_{\mathrm{G} / \mathrm{PGL}_{2}}^{0}(\mathbf{C}, E)=\emptyset$ if $g(\mathbf{C})=0$.

By functoriality of reduced Hurwitz spaces $[1, \S 6.2 .2]$, we have - corresponding to $\nu^{*}$ - a canonical morphism

$$
\nu: H_{\mathrm{G} / \mathrm{PGL}_{2}}^{0}(\mathbf{C}, E) \rightarrow H_{\mathrm{G} / \mathrm{PGL}_{2}}(\mathbf{C})
$$


Geometrically, $\nu$ corresponds to sending the $\mathrm{G} / \mathrm{PGL}_{2}$-isomorphism class of a Gcover $\tilde{f}: X \rightarrow \mathbb{P}^{1}$ with group $\tilde{E}$ to the $\mathrm{G} / \mathrm{PGL}_{2}$-isomorphism class of the Gcover $f: X \rightarrow X / G$ with group $G$. The defining condition $g\left(\mu^{*}(\tilde{\mathbf{C}})\right)=0$ in $H_{\mathrm{G} / \mathrm{PGL}_{2}}^{0}(\mathbf{C}, E)$ ensures that $X / G$ is isomorphic to $\mathbb{P}^{1}$.

Remark 4.2. There is no canonical choice for the isomorphism $X / G \simeq \mathbb{P}^{1}$ but the $\mathrm{G} / \mathrm{PGL}_{2}$-isomorphism class of $f$ is well and canonically defined from $\tilde{f}$. In other words, we have a diagram

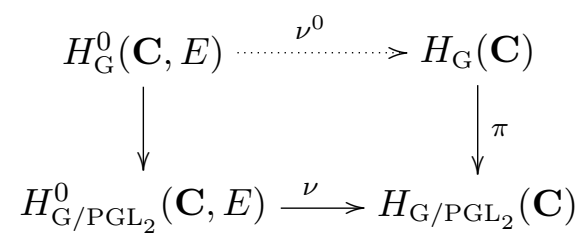

where $H_{\mathrm{G}}^{0}(\mathbf{C}, E)$ is defined by replacing $H_{\mathrm{G} / \mathrm{PGL}_{2}}(\tilde{\mathbf{C}})$ with $H_{\mathrm{G}}(\tilde{\mathbf{C}})$ in the definition of $H_{\mathrm{G} / \mathrm{PGL}_{2}}^{0}(\mathbf{C}, E)$. However, the lifting $\nu^{0}$ of $\nu$ does not exist, a priori.

As a result, we obtain a diagram of morphisms

$$
H_{\mathrm{G} / \mathrm{PGL}_{2}}^{0}(\mathbf{C}, E) \stackrel{\nu}{\longrightarrow} H_{\mathrm{G} / \mathrm{PGL}_{2}}(\mathbf{C}) \ll \pi
$$

Furthermore, we have

Lemma 4.3. The morphism $\nu: H_{\mathrm{G} / \mathrm{PGL}_{2}}^{0}(\mathbf{C}, E) \rightarrow H_{\mathrm{G} / \mathrm{PGL}_{2}}(\mathbf{C})$ is proper.

Proof. Given a finite group $G$, an integer $r \geq 3$ and a degree $r$ element $\mathbf{C} \in$ $\mathrm{R}_{+}^{*}(G)$, set $g=g(\mathbf{C}), d=|G|$ and $a=d(-2+r)-(2 g-2)$. Recall from section 2.2 that $H_{\mathrm{G} / \mathrm{PGL}_{2}}(\mathbf{C})$ is the Hurwitz scheme $H_{g, G, \mathbf{C}}$ of $[1]$.

Denote by $\phi: H_{g, G, \mathbf{C}} \rightarrow M_{g,[a]}$ the morphism corresponding to the stack morphism $\Phi$ sending a G-cover $f: X \rightarrow Y$ to the curve $X$ with the reduced ramification divisor. (Observe that the degree of this divisor is $a$.) Then, since $\phi$ is separated (as both $H_{g, G, \mathbf{C}}$ and $M_{g,[a]}$ are separated), it is enough to prove that $\psi=\phi \circ \nu: H_{\mathrm{G} / \mathrm{PGL}_{2}}^{0}(\mathbf{C}, E) \rightarrow M_{g,[a]}$ is proper. This, in turn, amounts to showing the valuative criterion of properness $[8$, Rem. 7.5.2] for the corresponding stack morphism $\Psi: \mathcal{H}_{\mathrm{G} / \mathrm{PGL}_{2}}^{0}(\mathbf{C}, E) \rightarrow \mathcal{M}_{g,[a]}{ }^{6}$. So let $R$ be a discrete valuation ring

\footnotetext{
${ }^{6}$ Indeed, this follows from the fact in general algebraic stack theory [5] that the natural morphism from a Deligne-Mumford stack to its coarse space is dominant and proper, together with the fact that $\psi$ is separated. (See [7, II, Ex. 4.4].)
} 
with field of fractions $K$ and consider any commutative diagram

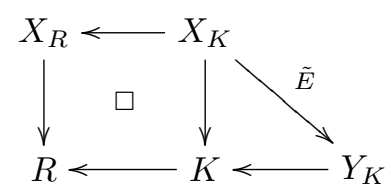

in which $X_{R}$ is a proper smooth curve of genus $g$ over $R$ and is equipped with a relatively étale divisor $D_{R}$ of degree $a$, such that $D_{K}$ is $\tilde{E}$-stable. Then we have to show that the action of $\tilde{E}$ on $\left(X_{K}, D_{K}\right)$ extends uniquely to $\left(X_{R}, D_{R}\right)$ and that the resulting monomorphism $\alpha: G \hookrightarrow \operatorname{Aut}\left(X_{R}\right)$ defines a G-cover $\left(X_{R} \rightarrow X_{R} / G, \alpha\right)$. But the former follows from the uniqueness of the stable model $\left(X_{R}, D_{R}\right)$ of the marked curve $\left(X_{K}, D_{K}\right)$ (cf. [1, Prop. 5.2]) and the latter is a consequence of $[1, \S 3.1]$. (Precisely speaking, $[1, \S 3.1]$ put the assumption $g \geq 2$, but this is superfluous. See also [15, Lem. 5.5])

Now, fix a finite group $G$, an integer $r \geq 3$ and a degree $r$ element $\mathbf{C} \in \mathrm{R}_{+}^{*}(G)$. Then, for any nontrivial conjugacy class $\mathcal{E}$ and any representative $E$ of $\mathcal{E}$, by construction, we have $\left.H_{\mathrm{G}}(\mathbf{C}, \supset \mathcal{E})=\varphi^{-1}\left(\nu\left(H_{\mathrm{G} / \mathrm{PGL}_{2}}^{0}(\mathbf{C}), E\right)\right)\right)$, which is a closed subset of $H_{\mathrm{G}}(\mathbf{C})$ since $\nu$ is proper. This yields theorem 1.2 as announced.

4.2.3. About the morphism $\nu$. By lemma 4.3, the morphism $\nu: H_{\mathrm{G} / \mathrm{PGL}_{2}}^{0}(\mathbf{C}, E) \rightarrow$ $H_{\mathrm{G} / \mathrm{PGL}_{2}}(\mathbf{C})$ is proper. But as $H_{\mathrm{G} / \mathrm{PGL}_{2}}^{0}(\mathbf{C}, E)$ and $H_{\mathrm{G} / \mathrm{PGL}_{2}}(\mathbf{C})$ are both affine schemes $\left(H_{\mathrm{G} / \mathrm{PGL}_{2}}(\mathbf{C})\right.$ is a finite cover of the affine scheme $\left.M_{0,[r]}\right)$, it results from [9, Lem. 3.3.17] that $\nu$ is finite. By [9, Ex. 5.1.25], to compute its degree, it is enough to compute the cardinality of its geometric fibers.

The aim of this section is to describe the geometrically irreducible components of $H_{\mathrm{G} / \mathrm{PGL}_{2}}(\mathbf{C}, \supset \mathcal{E})$ for finite groups $G$ with trivial center, where $\mathcal{E}$ is the conjugacy class of $E=C_{n}(n \geq 6), D_{2 n}(2 \mid n, n \geq 6), \mathcal{S}_{4}, \mathcal{A}_{5}$. It will appear in the course of the proof (lemma 4.7) why our method does not seem to work for other groups $E$.

More precisely, since $G$ has trivial center, it follows from remark (1) of section 4.1 that the only possible extensions $1 \rightarrow G \rightarrow \tilde{E} \rightarrow E \rightarrow 1$ are the trivial split extensions $\tilde{E}=G \times E$. Let us write $p_{G}: G \times E \rightarrow G$ and $p_{E}: G \times E \rightarrow E$ for the canonical projections, $\mu_{G}^{*}: \mathrm{R}_{+}(G \times E) \rightarrow \mathrm{R}_{+}^{*}(G)$ and $\mu_{E}^{*}: \mathrm{R}_{+}(G \times E) \rightarrow \mathrm{R}_{+}^{*}(E)$ for the corresponding morphisms of monoids defined in section 4.2.1. Similarly, 
write $i_{G}: G \hookrightarrow G \times E$ and $i_{E}: E \hookrightarrow G \times E$ for the canonical embeddings, $\nu_{G}^{*}: \mathrm{R}_{+}(G \times E) \rightarrow \mathrm{R}_{+}^{*}(G)$ and $\nu_{E}^{*}: \mathrm{R}_{+}(G \times E) \rightarrow \mathrm{R}_{+}^{*}(E)$ for the corresponding morphisms of monoids.

To proceed, set

$$
\operatorname{Aut}^{G}(G \times E):=\left\{\tilde{\beta} \in \operatorname{Aut}(G \times E) \mid \tilde{\beta} \circ i_{G}=i_{G}\right\} .
$$

Then we have two natural homomorphisms

$$
a: \operatorname{Aut}(E) \rightarrow \operatorname{Aut}^{G}(G \times E), \beta \mapsto \operatorname{Id}_{G} \times \beta
$$

and

$$
b: \operatorname{Aut}^{G}(G \times E) \rightarrow \operatorname{Aut}(E), \tilde{\beta} \mapsto \bar{\beta},
$$

where $\overline{\tilde{\beta}}$ denotes the automorphism that $\tilde{\beta}$ induces on the quotient $E=(G \times$ $E) /(G \times\{1\})$, or, equivalently, $\overline{\tilde{\beta}}$ is characterized by: $\overline{\tilde{\beta}} \circ p_{E}=p_{E} \circ \tilde{\beta}$.

Lemma 4.4. (Assuming that $G$ has trivial center.) The homomorphisms a and $b$ are inverse to each other. In particular, $\operatorname{Aut}(E) \simeq \operatorname{Aut}^{G}(G \times E)$.

Proof. It is trivial that $b \circ a=\mathrm{Id}$. To see that $a \circ b=\mathrm{Id}$, take any $\tilde{\beta} \in \mathrm{Aut}^{G}(G \times E)$ and compare $\tilde{\beta}$ and $(b \circ a)(\tilde{\beta})=\operatorname{Id}_{G} \times \overline{\tilde{\beta}}$. Since $G$ has trivial center, the subgroup $\{1\} \times E$ coincides with the centralizer of $G \times\{1\}$, hence is preserved by $\tilde{\beta}$. Now, since the subgroup $\{1\} \times E$ is isomorphically mapped onto the quotient $E=(G \times E) /(G \times\{1\}), \tilde{\beta}$ must induce $\overline{\tilde{\beta}}$ on the subgroup $\{1\} \times E$. Thus, $\tilde{\beta}$ and $\operatorname{Id}_{G} \times \overline{\tilde{\beta}}$ coincide with each other both on $G \times\{1\}$ and on $\{1\} \times E$. This completes the proof, since $G \times E$ is generated by $G \times\{1\}$ and $\{1\} \times E$.

Note that $a$ and $b$ induce an isomorphism $\operatorname{Out}(E) \simeq \operatorname{Out}^{G}(G \times E)$, where $\operatorname{Out}(E):=\operatorname{Aut}(E) / \operatorname{Inn}(E)$ and $\operatorname{Out}^{G}(G \times E):=\operatorname{Aut}^{G}(G \times E) / \operatorname{Inn}(\{1\} \times E)$.

Next, let $\mathcal{X}(\mathbf{C}, E)$ denote the set of all $\tilde{\mathbf{C}} \in\left(\nu_{G}^{*}\right)^{-1}(\mathbf{C}) \cap \mathrm{R}_{+}^{*}(G \times E)$ such that $g\left(\mu_{E}^{*}(\tilde{\mathbf{C}})\right)=0$. Then $\operatorname{Aut}^{G}(G \times E)$ acts naturally on $\mathcal{X}(\mathbf{C}, E) \subset \mathrm{R}_{+}^{*}(G \times E)$ (by pulling-back). Moreover, compatibly with this action, $\operatorname{Aut}^{G}(G \times E)$ also acts naturally on $\coprod_{\tilde{\mathbf{C}} \in \mathcal{X}(\mathbf{C}, E)} H_{\mathrm{G} / \mathrm{PGL}_{2}}(\tilde{\mathbf{C}})$, by sending, for each $\tilde{\beta} \in \operatorname{Aut}^{G}(G \times E)$, a G-curve $(\tilde{X}, j: G \times E \hookrightarrow \operatorname{Aut}(\tilde{X}))$ to $(\tilde{X}, j \circ \tilde{\beta}: G \times E \hookrightarrow \operatorname{Aut}(\tilde{X}))$.

Lemma 4.5. The actions of $\operatorname{Aut}^{G}(G \times E)$ on $\mathcal{X}(\mathbf{C}, E)$ and on $\coprod_{\tilde{\mathbf{C}} \in \mathcal{X}(\mathbf{C}, E)} H_{\mathrm{G} / \mathrm{PGL}_{2}}(\tilde{\mathbf{C}})$ factor through $\operatorname{Aut}^{G}(G \times E) \rightarrow \operatorname{Out}^{G}(G \times E)$. 
Proof. As $\operatorname{Inn}(G \times E)$ acts trivially on $\mathrm{R}_{+}^{*}(G \times E)$, $\operatorname{Inn}(\{1\} \times E)$ acts trivially on $\mathcal{X}(\mathbf{C}, E)$. Next, let $(\tilde{X}, j: G \times E \hookrightarrow \operatorname{Aut}(\tilde{X}))$ be a G-curve corresponding to a point of $\coprod_{\tilde{\mathbf{C}} \in \mathcal{X}(\mathbf{C}, E)} H_{\mathrm{G} / \mathrm{PGL}_{2}}(\tilde{\mathbf{C}})$, and $\tilde{\beta}=\operatorname{Inn}((1, e)) \in \operatorname{Inn}(\{1\} \times E)$, where $e \in E$. Then, $j((1, e))$ tautologically gives a G-curve isomorphism from $(\tilde{X}, j)$ to $(\tilde{X}, j \circ \tilde{\beta})$. Thus, the action of $\tilde{\beta}$ on $\coprod_{\tilde{\mathbf{C}} \in \mathcal{X}(\mathbf{C}, E)} H_{\mathrm{G}^{\prime} \mathrm{PGL}_{2}}(\tilde{\mathbf{C}})$ is trivial, as desired.

Denote by $\sim$ the equivalence relation on $\mathcal{X}(\mathbf{C}, E)$ induced by the action of $\operatorname{Out}^{G}(G \times E)$. Also, for each $\tilde{\mathbf{C}} \in \mathcal{X}(\mathbf{C}, E)$, denote by $\mathrm{Out}^{G}(G \times E)_{\tilde{\mathbf{C}}}$ the stabilizer subgroup of $\mathrm{Out}^{G}(G \times E)$ at $\tilde{\mathbf{C}}$.

Proposition 4.6. Let $\mathcal{E}$ be the conjugacy class in $\mathrm{PGL}_{2}(\bar{k})$ of one of the following groups: $C_{n}, n \geq 6 ; D_{2 n}, 2 \mid n, n \geq 6 ; \mathcal{S}_{4} ; \mathcal{A}_{5}$ (cf. lemma 1.1), and $E$ a representative of $\mathcal{E}$. Let $G$ be a finite group with trivial center. Then the finite morphism $\nu_{G}: \coprod_{\tilde{\mathbf{C}} \in \mathcal{X}(\mathbf{C}, E)} H_{\mathrm{G} / \mathrm{PGL}_{2}}(\tilde{\mathbf{C}}) \rightarrow H_{\mathrm{G} / \mathrm{PGL}_{2}}(\mathbf{C})$ induces a finite, radicial (i.e., universally injective) morphism

$$
\left(\coprod_{\tilde{\mathbf{C}} \in \mathcal{X}(\mathbf{C}, E)} H_{\mathrm{G} / \mathrm{PGL}_{2}}(\tilde{\mathbf{C}})\right) / \mathrm{Out}^{G}(G \times E) \rightarrow H_{\mathrm{G} / \mathrm{PGL}_{2}}(\mathbf{C})
$$

with image $H_{\mathrm{G} / \mathrm{PGL}_{2}}(\mathbf{C}, \supset \mathcal{E})$. Moreover, the left-hand side is naturally identified with $\coprod_{\tilde{\mathbf{C}} \in \mathcal{X}(\mathbf{C}, E) / \sim}\left(H_{\mathrm{G} / \mathrm{PGL}_{2}}(\tilde{\mathbf{C}}) / \mathrm{Out}^{G}(G \times E)_{\tilde{\mathbf{C}}}\right)$. In particular, the geometrically irreducible components of $H_{\mathrm{G} / \mathrm{PGL}_{2}}(\mathbf{C}, \supset \mathcal{E})$ can be identified with those of $\coprod_{\tilde{\mathbf{C}} \in \mathcal{X}(\mathbf{C}, E) / \sim}\left(H_{\mathrm{G} / \mathrm{PGL}_{2}}(\tilde{\mathbf{C}}) / \mathrm{Out}^{G}(G \times E)_{\tilde{\mathbf{C}}}\right)$.

For any $\tilde{\mathbf{C}}$ with $H_{\mathrm{G} / \mathrm{PGL}_{2}}(\tilde{\mathbf{C}}) \neq \emptyset$, the group $\mathrm{Out}^{G}(G \times E)_{\tilde{\mathbf{C}}}$ is trivial for $\mathcal{E} \sim \mathcal{S}_{4}$, $\mathcal{A}_{5}$ and of order at most 2 (depending on $\tilde{\mathbf{C}}$ ) for $\mathcal{E} \sim C_{n}(n \geq 6), D_{2 n}(2 \mid n, n \geq$ 6). In particular, if $\mathcal{E} \sim \mathcal{S}_{4}, \mathcal{A}_{5}$, then the geometrically irreducible components of $H_{\mathrm{G} / \mathrm{PGL}_{2}}(\mathbf{C}, \supset \mathcal{E})$ can be identified with those of $\coprod H_{\mathrm{G} / \mathrm{PGL}_{2}}(\tilde{\mathbf{C}})^{7}$. $\tilde{\mathbf{C}} \in \mathcal{X}(\mathbf{C}, E) / \sim$

Proof. Let us start from $\tilde{\mathbf{C}}_{i} \in \mathcal{X}(\mathbf{C}, E), i=1,2$. For each $i=1,2$, let $\left(\tilde{X}_{i}, j_{i}\right.$ : $\left.G \times E \hookrightarrow \operatorname{Aut}\left(\tilde{X}_{i}\right)\right)$ be a G-curve with group $G \times E$ corresponding to an element

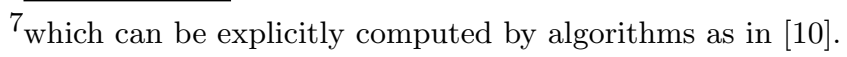


of $H_{\mathrm{G} / \mathrm{PGL}_{2}}\left(\tilde{\mathbf{C}}_{i}\right)(\bar{k})$ and define a G-curve $\left(X_{i}, i_{i}\right)$ with group $E$ to be $\mu_{E}\left(\left(\tilde{X}_{i}, j_{i}\right)\right)$, or, more precisely, $X_{i}=\tilde{X}_{i} /\left(j_{i} \circ i_{G}\right)(G)$ and $i_{i}: E \hookrightarrow \operatorname{Aut}\left(X_{i}\right)$ is defined by $f_{i} \circ j_{i}(\tilde{e})=\left(i_{i} \circ p_{E}\right)(\tilde{e}) \circ f_{i}, \tilde{e} \in G \times E$, where $f_{i}: \tilde{X}_{i} \rightarrow X_{i}$ denotes the G-cover corresponding to the G-curve $\left(\tilde{X}_{i}, j_{i} \circ i_{G}\right)=\nu_{G}\left(\left(\tilde{X}_{i}, j_{i}\right)\right)$ with group $G$. We have to show that, for $E$ as in proposition $4.6,\left(\tilde{X}_{1}, j_{1} \circ i_{G}\right) \simeq\left(\tilde{X}_{2}, j_{2} \circ i_{G}\right)$ implies $\left(\tilde{X}_{1}, j_{1} \circ \tilde{\beta}\right) \simeq\left(\tilde{X}_{2}, j_{2}\right)$ for some $\tilde{\beta} \in \operatorname{Aut}^{G}(G \times E)$. So, fix a G-curve isomorphism $\tilde{v}:\left(\tilde{X}_{1}, j_{1} \circ i_{G}\right) \tilde{\rightarrow}\left(\tilde{X}_{2}, j_{2} \circ i_{G}\right)$, or, equivalently, a weak G-isomorphism:

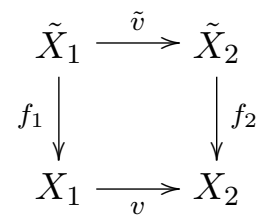

Eventually, write $\Pi_{i}: X_{i} \rightarrow B_{i}$ for the G-cover corresponding to the G-curve $\left(X_{i}, i_{i}\right)$ with group $E$. The following commutative diagram sums up the notation we will use:

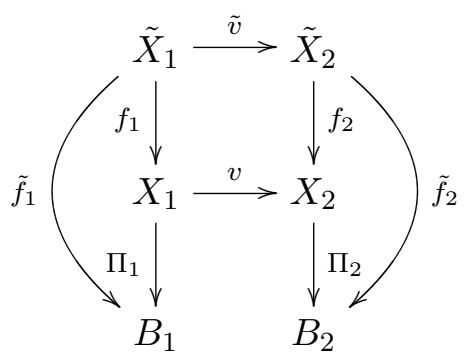

Note that $X_{i} \simeq B_{i} \simeq \mathbb{P}_{\bar{k}}^{1}, i=1,2$.

Before going on, we need to introduce one more notion. We say that a finite subgroup $E$ of $\mathrm{PGL}_{2}(\bar{k})$ has property $(*)$ if there is no finite subgroup $E^{\prime}$ of $\mathrm{PGL}_{2}(\bar{k})$ which contains two distinct subgroups isomorphic to $E$ (or, equivalently, two distinct conjugates of $E$ ). We leave it as an easy exercise to the reader to check the following:

Lemma 4.7. Let $E$ be a nontrivial finite subgroup of $\mathrm{PGL}_{2}(\bar{k})$. Then $E$ has property (*) if and only if it is of the form $C_{n}, n \geq 6, D_{2 n}, 2 \mid n, n \geq 6, \mathcal{S}_{4}, \mathcal{A}_{5}$.

From now on, we assume that $E$ is a group of the form of those listed in lemma 4.7. Let $E_{i} \subset \operatorname{Aut}\left(X_{i}\right)$ denote the base group of $f_{i}, i=1,2$. Then, on the one hand, $i_{i}(E) \subset E_{i}, i=1,2$, and, on the other hand, $v E_{1} v^{-1}=E_{2}$. Hence, according to property $(*)$, we get $v i_{1}(E) v^{-1}=i_{2}(E)$. It follows from this 
last equality that the isomorphism $v: X_{1} \rightarrow X_{2}$ induces a unique isomorphism $b: B_{1} \rightarrow B_{2}$ that makes the following diagram commutative:

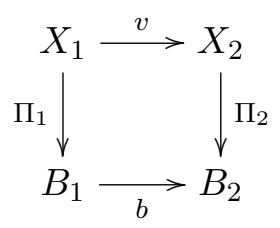

Now, the whole diagram becomes

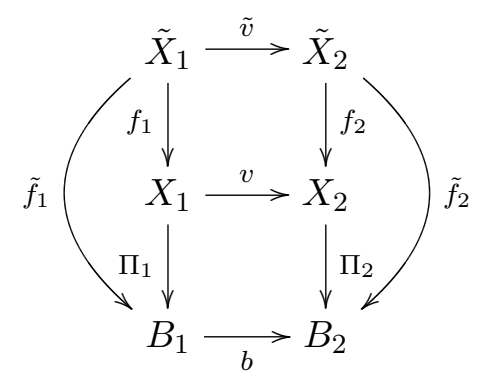

Here, note that $(\tilde{v}, b)$ does not necessarily give a weak G-isomorphism between $\tilde{f}_{1}: \tilde{X}_{1} \rightarrow B_{1}$ and $\tilde{f}_{2}: \tilde{X}_{2} \rightarrow B_{2}$, as $(v, b)$ does not necessarily give a weak G-isomorphism between $\Pi_{1}: X_{1} \rightarrow B_{1}$ and $\Pi_{2}: X_{2} \rightarrow B_{2}$. To adjust this, note that we have two isomorphisms $j_{2}, j_{2}^{\prime}: G \times E \rightarrow \operatorname{Aut}\left(\tilde{X}_{2} / B_{2}\right)$, where $j_{2}^{\prime}(\tilde{e}):=\tilde{v} j_{1}(\tilde{e}) \tilde{v}^{-1}, \tilde{e} \in G \times E$. So, set $\tilde{\beta}:=\left(j_{2}^{\prime}\right)^{-1} \circ j_{2} \in \operatorname{Aut}(G \times E)$. The fact that $(\tilde{v}, v)$ is a weak G-isomorphism implies that $\tilde{\beta} \in \operatorname{Aut}^{G}(G \times E)$. Now, the G-curve $\left(\tilde{X}_{1}, j_{1} \circ \tilde{\beta}\right)$ is isomorphic (via $\left.\tilde{v}\right)$ to $\left(\tilde{X}_{2}, j_{2}^{\prime} \circ \tilde{\beta}\right)=\left(\tilde{X}_{2}, j_{2}\right)$. This completes the proof of the first assertion.

Next, the identification

$$
\left(\coprod_{\tilde{\mathbf{C}} \in \mathcal{X}(\mathbf{C}, E)} H_{\mathrm{G} / \mathrm{PGL}_{2}}(\tilde{\mathbf{C}})\right) / \mathrm{Out}^{G}(G \times E)=\coprod_{\tilde{\mathbf{C}} \in \mathcal{X}(\mathbf{C}, E) / \sim}\left(H_{\mathrm{G} / \mathrm{PGL}_{2}}(\tilde{\mathbf{C}}) / \mathrm{Out}^{G}(G \times E)_{\tilde{\mathbf{C}}}\right)
$$

just follows from elementary theory of group action.

Eventually, we shall analyze the group $\operatorname{Out}^{G}(G \times E)_{\tilde{\mathbf{C}}}$, under the assumption that $H_{\mathrm{G} / \mathrm{PGL}_{2}}(\tilde{\mathbf{C}}) \neq \emptyset$. Set $\boldsymbol{\Gamma}:=\mu_{E}^{*}(\tilde{\mathbf{C}})$, which must be realized as the inertia canonical invariant of a G-cover $\Pi: X \rightarrow B$ with group $E$ and with $X \simeq B \simeq \mathbb{P} \frac{1}{k}$. Now, the Riemann-Hurwitz formula gives the possible ramification indices of $\Pi$ and the possible values for the inertia canonical invariant $\boldsymbol{\Gamma}$ of $\Pi$. With the 
notation of [4], we have:

(3)

\begin{tabular}{|c|c|c|}
\hline$E$ & Ramification indices & Inertia canonical invariant \\
\hline $\mathcal{A}_{5}$ & $(2,3,5)$ & $(2 A, 3 A, 5 A)$ or $(2 A, 3 A, 5 B)$ \\
\hline $\mathcal{S}_{4}$ & $(2,3,4)$ & $(2 A, 3 A, 4 A)$ \\
\hline $\mathcal{A}_{4}$ & $(2,3,3)$ & $(2 A, 3 A, 3 B)$ \\
\hline$D_{4}$ & $(2,2,2)$ & $(2 A, 2 B, 2 C)$ \\
\hline$D_{2 n}, n \geq 3$ & $(2,2, n)$ & $\left(2 A, 2 A,(n A)^{k}\right),(k, n)=1$ if $2 \nmid n$ \\
& & $\left(2 A, 2 B,(n A)^{k}\right),(k, n)=1$ if $2 \mid n$ \\
\hline$C_{n}, n \geq 2$ & $(n, n)$ & $\left((n A)^{k},(n A)^{n-k}\right),(k, n)=1$ \\
\hline
\end{tabular}

(For the sake of completeness, we have included all the possible groups in lemma 1.1, not only the ones appearing in proposition 4.6.)

A straightforward computation [13, Chap. 7] shows that the tuples in table 3 are all "rigid", that is, given such $E, \boldsymbol{\Gamma}=\left(\Gamma_{1}, \ldots, \Gamma_{s}\right)(s:=\operatorname{deg}(\boldsymbol{\Gamma}))$, and a ramification divisor $\mathbf{t}=t_{1}+\cdots+t_{s} \subset B\left(\simeq \mathbb{P} \frac{1}{k}\right)$ (ordered) of degree $s$, there is a unique G-isomorphism class of G-cover $X \rightarrow B$ with group $E$, ramification divisor $\mathbf{t}$ and inertia canonical invariant $\boldsymbol{\Gamma}$ (with $\Gamma_{i}$ corresponding to $t_{i}, i=1, \ldots, s$ ). In particular, if $\beta \in \operatorname{Aut}(E)$ satisfies $\beta^{*}\left(\Gamma_{i}\right)=\Gamma_{i}, i=1, \ldots, s$, then $\beta$ is an inner automorphism.

Now, take any $\tilde{\beta}$ such that $\tilde{\beta}^{*}(\tilde{\mathbf{C}})=\tilde{\mathbf{C}}$ and set $\beta:=\overline{\tilde{\beta}} \in \operatorname{Aut}(E)$. Then we have $\beta^{*}(\boldsymbol{\Gamma})=\boldsymbol{\Gamma}$. This, together with the above rigidity, implies that the natural homomorphism from $\mathrm{Out}^{G}(G \times E)_{\tilde{\mathbf{C}}}$ to the symmetric group on the conjugacy classes $\left\{\Gamma_{1}, \ldots, \Gamma_{s}\right\}$ is injective. Further, by observing $o\left(\beta^{*}\left(\Gamma_{i}\right)\right)=o\left(\Gamma_{i}\right), i=1, \ldots, s$, we see that the image of this last injective homomorphism is trivial for $E \sim \mathcal{S}_{4}$, $\mathcal{A}_{5}$ and is contained in a group of order 2 for $E \sim C_{n}(n \geq 6), D_{2 n}(2 \mid n, n \geq 6)$. This completes the proof.

\section{Application to the Regular inverse Galois problem over $\mathbb{Q}$}

We retain the notation of section 4.2. The regular inverse Galois problem over $\mathbb{Q}$ amounts to finding $\mathbb{Q}$-rational points on non-reduced Hurwitz spaces [6]. One way to do this is to search for $\mathbb{Q}$-rational points on reduced Hurwitz spaces and, then, try and lift these $\mathbb{Q}$-rational points from reduced to non-reduced Hurwitz spaces. One result of [3] is the following. Let $\mathcal{E}$ be the conjugacy class of a 
nontrivial finite subgroup of $\mathrm{PGL}_{2}(\overline{\mathbb{Q}})$ and set $d(\mathcal{E})=1$ if $\mathcal{E} \sim \mathcal{A}_{5}, \mathcal{S}_{4}$ or $D_{2 n}$, $n \geq 3, d(\mathcal{E})=2$ if $\mathcal{E} \sim \mathcal{A}_{4}$ or $C_{n}, n \geq 2$ and $d(\mathcal{E})=6$ if $\mathcal{E} \sim D_{4}$.

Proposition 5.1. Any closed point $p \in H_{\mathrm{G} / \mathrm{PGL}_{2}}(\mathbf{C}, \mathcal{E})$ admits a lift $\mathbf{p} \in H_{\mathrm{G}}(\mathbf{C}, \mathcal{E})$ such that $[\kappa(\mathbf{p}): \kappa(p)] \leq d(\mathcal{E})$.

In other words, the most interesting strata on reduced Hurwitz spaces are those corresponding to $\mathcal{E} \sim \mathcal{A}_{5}, \mathcal{S}_{4}$ or $D_{2 n}, n \geq 3$. But the main problem remains: finding $\mathbb{Q}$-rational points on these strata; except for $r=3,4[3, \S 5]$, we currently have no method to do this.

The idea of the method presented here is to reverse this process, that is, starting from a reduced Hurwitz space $H_{\mathrm{G} / \mathrm{PGL}_{2}}(\mathbf{C})$ with a $\mathbb{Q}$-rational point (which does not lift, a priori, to a $\mathbb{Q}$-rational point on $H_{\mathrm{G}}(\mathbf{C})$ ), send this $\mathbb{Q}$-rational point to a $\mathbb{Q}$-rational point on a reduced Hurwitz space in a stratum $H_{\mathrm{G} / \mathrm{PGL}_{2}}(\mathbf{C}(\mathcal{E}), \mathcal{E})$ (where $\mathbf{C}(\mathcal{E})$ is an inertia canonical invariant to be explicitly defined), with $\mathcal{E} \sim$ $\mathcal{A}_{5}, \mathcal{S}_{4}, D_{2 n}, n \geq 3$.

Now, let $\boldsymbol{\Gamma} \in \mathrm{R}_{+}^{*}(E)$ be one of the tuples in table 3 and choose $\tilde{\mathbf{C}} \in \mu_{G}^{-1}(\mathbf{C}) \cap$ $\left(\mu_{E}^{*}\right)^{-1}(\boldsymbol{\Gamma}) \cap \mathrm{R}_{+}^{*}(G \times E) .{ }^{8}$ Then the rigidity of $\boldsymbol{\Gamma}$ forces a certain relative rigidity of $\tilde{\mathbf{C}}$ over $\mathbf{C}$. In terms of Hurwitz spaces, this latter rigidity shows that the natural morphism $\mu_{G}: H_{\mathrm{G} \mathrm{PGL}_{2}}(\tilde{\mathbf{C}}) \rightarrow H_{\mathrm{G}_{\mathrm{PGL}}}(\mathbf{C})$ (defined over $\mathbb{Q}_{\tilde{\mathbf{C}}}$ ) is radicial. We thus obtain the following diagram defined over $\mathbb{Q}_{\tilde{\mathbf{C}}}$ :

$$
\begin{gathered}
H_{\mathrm{G} / \mathrm{PGL}_{2}}(\mathbf{C}) \longleftarrow \mu_{G} \\
H_{\mathrm{G} / \mathrm{PGL}_{2}}(\tilde{\mathbf{C}}) \\
\qquad \nu \\
\quad H_{\mathrm{G} / \mathrm{PGL}_{2}}\left(\nu_{G}^{*}(\tilde{\mathbf{C}}), \supset \mathcal{E}\right) \longleftarrow{ }^{\varphi} H_{\mathrm{G}}\left(\nu_{G}^{*}(\tilde{\mathbf{C}}), \supset \mathcal{E}\right)
\end{gathered}
$$

For $\mathcal{E} \sim \mathcal{A}_{5}$ or $\mathcal{S}_{4}$, one has $H_{\mathrm{G}}\left(\nu_{G}^{*}(\tilde{\mathbf{C}}), \supset \mathcal{E}\right)=H_{\mathrm{G}}\left(\nu_{G}^{*}(\tilde{\mathbf{C}}), \mathcal{E}\right)$, and, for $\mathcal{E} \sim D_{2 n}$, $n \geq 3$, one can write $H_{\mathrm{G}}\left(\nu_{G}^{*}(\tilde{\mathbf{C}}), \supset \mathcal{E}\right)$ as a disjoint union of strata $H_{\mathrm{G}}\left(\nu_{G}^{*}(\tilde{\mathbf{C}}), \mathcal{E}\right)$ with $\mathcal{E} \sim \mathcal{S}_{4}$ (if $n=3,4$ ) or $D_{2 m}, n \mid m$. Thus, we get:

Corollary 5.2. Assume that $\mathcal{E} \sim \mathcal{A}_{5}, \mathcal{S}_{4}$ or $D_{2 n}, n \geq 3$ and that $\mu_{G}: H_{\mathrm{G} / \mathrm{PGL}_{2}}(\tilde{\mathbf{C}}) \rightarrow$ $H_{\mathrm{G} / \mathrm{PGL}_{2}}(\mathbf{C})$ is surjective (hence universally bijective). If $H_{\mathrm{G} / \mathrm{PGL}_{2}}(\mathbf{C})\left(\mathbb{Q}_{\mathbf{C}}\right) \neq \emptyset$, then $H_{\mathrm{G}}\left(\nu_{G}^{*}(\tilde{\mathbf{C}})\right)\left(\mathbb{Q}_{\tilde{\mathbf{C}}}\right) \neq \emptyset$.

\footnotetext{
$\overline{8 \text { Any orderings }} \mathbf{C}=\left(C_{1}, \ldots, C_{r}\right), \boldsymbol{\Gamma}=\left(\Gamma_{1}, \ldots, \Gamma_{s}\right)$ (with $\left.r \geq 3, s=2,3\right)$ define such an element by setting $\tilde{\mathbf{C}}=\sum_{1 \leq i \leq r} C_{i} \times \Gamma_{i} \in \mathrm{R}_{+}^{*}(G \times E)$, and conversely. (Here, we set $\Gamma_{i}=\{1\}$ for $s<i \leq r$.) In particular, the degree of $\tilde{\mathbf{C}}$ is $r$.
} 
In the table below, we give an example of tuples $\nu_{G}^{*}(\tilde{\mathbf{C}})$ for the orderings $\mathbf{C}=\left(C_{1}, \ldots, C_{r}\right), \boldsymbol{\Gamma}=(2 A, 3 A, 5 A)$ or $(2 A, 3 A, 5 B), \boldsymbol{\Gamma}=(2 A, 3 A, 4 A)$ and $\boldsymbol{\Gamma}=\left(2 A, 2 A,(n A)^{k}\right)(2 \bigwedge n,(k, n)=1) /\left(2 A, 2 B,(n A)^{k}\right)(2 \mid n,(k, n)=1)$ if $E=\mathcal{A}_{5}, \mathcal{S}_{4}$ and $D_{2 n}, n \geq 3$ respectively.

\begin{tabular}{|c|c|}
\hline$E$ & inertia canonical invariant $\nu_{G}^{*}(\tilde{\mathbf{C}})$ \\
\hline $\mathcal{A}_{5}$ & $30 C_{1}^{2}+20 C_{2}^{3}+12 C_{3}^{5}+60\left(C_{4}+\cdots+C_{r}\right)$ \\
\hline $\mathcal{S}_{4}$ & $12 C_{1}^{2}+8 C_{2}^{3}+6 C_{3}^{4}+24\left(C_{4}+\cdots+C_{r}\right)$ \\
\hline$D_{2 n}, n \geq 3$ & $n\left(C_{1}^{2}+C_{2}^{2}\right)+2 C_{3}^{n}+2 n\left(C_{4}+\cdots+C_{r}\right)$ \\
\hline
\end{tabular}

Note that $\mathbb{Q}_{\boldsymbol{\Gamma}}=\mathbb{Q}(\sqrt{5}), \mathbb{Q}, \mathbb{Q}\left(\zeta_{n}+\zeta_{n}^{-1}\right)$ for $\mathcal{E} \sim \mathcal{A}_{5}, \mathcal{S}_{4}$ or $D_{2 n}, n \geq 3$ respectively, hence the cases $\mathcal{E} \sim \mathcal{S}_{4}, D_{2 n}, n=3,4,6$, are only worth considering when searching for $\mathbb{Q}$-rational points.

Remark 5.3. Checking the surjectivity of $\mu_{G}$ amounts to the following purely group-theoretic problem. Given a finite group $G$ and an $r$-tuple $\mathbf{C}=\left(C_{1}, \ldots, C_{r}\right)$ of nontrivial conjugacy classes in $\mathbf{C}$, let $\operatorname{ni}_{G}(\mathbf{C})$ denote the corresponding Nielsen class, that is, the set of all $r$-tuples $\mathbf{g}=\left(g_{1}, \ldots, g_{r}\right) \in G^{r}$ modulo diagonal componentwise inner conjugation by elements of $G$ such that (i) $g_{1}, \ldots, g_{r}$ generate $G$, (ii) $g_{i} \in C_{\sigma(i)}, i=1, \ldots, r$ for some permutation $\sigma \in \mathcal{S}_{r}$ and (iii) $g_{1} \cdots g_{r}=1$. Write $\boldsymbol{\Gamma}=\left(\Gamma_{1}, \ldots, \Gamma_{s}\right)$ and $\tilde{\mathbf{C}}=\left(C_{1} \times \Gamma_{1}, \ldots, C_{r} \times \Gamma_{r}\right)$ (where we set $\Gamma_{i}=\{1\}$ for $\left.s<i \leq r\right)$. Then $\mu_{G}$ is surjective if and only if any element $\mathbf{g}=\left(g_{1}, \ldots, g_{r}\right) \in \operatorname{ni}_{G}(\mathbf{C})$ lifts to some element $\tilde{\mathbf{e}} \in \mathrm{ni}_{\tilde{E}}(\tilde{\mathbf{C}})$. If such a lift exists, by rigidity, it is $\tilde{\mathbf{e}}=\left(\left(g_{1}, \gamma_{1}\right),\left(g_{2}, \gamma_{2}\right),\left(g_{3}, \gamma_{3}\right),\left(g_{4}, 1\right), \ldots,\left(g_{r}, 1\right)\right)$, where $\left(\gamma_{1}, \gamma_{2}, \gamma_{3}\right)$ denotes the unique element of $\operatorname{ni}_{E}(\boldsymbol{\Gamma})$ with $\gamma_{i} \in \Gamma_{i}$. The only difficulty is, thus, to ensure that $\tilde{e}_{1}, \ldots, \tilde{e}_{r}$ generate $\tilde{E}$. This does not hold in general. (Take for instance $G=E$ and $\mathbf{C}=\boldsymbol{\Gamma}$. Then $\tilde{e}_{1}, \ldots, \tilde{e}_{s}$ only generate (a conjugate of) the diagonal subgroup of $\tilde{E}=E \times E$.) But one can give several sufficient conditions for this to hold. An easy-to-check one (see example 5.4 below) is:

Criterion. If $\operatorname{gcd}\left(o\left(C_{i}\right), o\left(\Gamma_{i}\right)\right)=1$ for at least $s-1$ of the $s$ indices $i=1, \ldots, s$, then $\mu_{G}$ is surjective.

Indeed, assume, for instance, that $s=3$ and that $\operatorname{gcd}\left(o\left(C_{i}\right), o\left(\Gamma_{i}\right)\right)=1$ for $i=2,3$. Then $\tilde{e}_{2}^{o\left(\Gamma_{2}\right)}, \tilde{e}_{3}^{o\left(\Gamma_{3}\right)}, \tilde{e}_{4}, \ldots, \tilde{e}_{r}$ generate $G \times\{1\} \subset \tilde{E}=G \times E$, since $g_{2}^{o\left(\Gamma_{2}\right)}, g_{3}^{o\left(\Gamma_{3}\right)}, g_{4}, \ldots, g_{r}$ generate $G$. (Recall that $g_{1}=\left(g_{2} \cdots g_{r}\right)^{-1}$.) 
Example 5.4. [3, Ex.5.8] Consider $G=L_{2}(25), \mathbf{C}=4(3 A)$ and note that $3 A$ is a $\mathbb{Q}$-rational conjugacy class. Regarding the natural compactification $\bar{H}_{\mathrm{G} / \mathrm{PGL}_{2}}(\mathbf{C})$ as a cover $\bar{H}_{\mathrm{G} / \mathrm{PGL}_{2}}(\mathbf{C}) \rightarrow \mathbb{P}^{1}$ ramified above $0,1, \infty$, we obtain [10] the following list for the lengths of the usual/reduced orbits and the corresponding reduced genera

$$
(1200 / 300,7),(936 / 468,17),(304 / 304,5),(120 / 30,0) .
$$

The unique reduced compactified orbit $\bar{O}_{\mathrm{G} / \mathrm{PGL}_{2}}$ of length 30 and reduced genus 0 has ramification type:

$$
\begin{array}{ll}
\text { Above } 0 & :\left[(3)^{10}\right] \\
\text { Above } 1 & :\left[(2)^{15}\right] \\
\text { Above } \infty & :\left[(2)^{2},(3)^{2},(6)^{2},(8)^{1}\right]
\end{array}
$$

In particular $\bar{O}_{\mathrm{G} / \mathrm{PGL}_{2}}$ carries a $\mathbb{Q}$-rational ramified point (the one with ramification index 8) above $\infty$ so $O_{\mathrm{G} / \mathrm{PGL}_{2}}$ is birational to $\mathbb{P}_{\mathbb{Q}}^{1}$ over $\mathbb{Q}$ and, hence, $H_{\mathrm{G} / \mathrm{PGL}_{2}}(\mathbf{C})(\mathbb{Q}) \neq \emptyset$. However, the G-covers classified by $O_{\mathrm{G} / \mathrm{PGL}_{2}}$ have trivial base invariant $[3, \S 5]$ so proposition 5.1 cannot be applied to assert that $O_{\mathrm{G}}(\mathbb{Q}) \neq \emptyset$. But since $3 A$ is a $\mathbb{Q}$-rational conjugacy class, corollary 5.2 can be applied with $\mathcal{E} \sim \mathcal{S}_{4}$ yields a regular realization of $L_{2}(25)$ over $\mathbb{Q}$ with inertia canonical invariant $42(3 A)$.

\section{Acknowledgments}

The authors would like to thank Seidai Yasuda for suggesting the improvement of the first approach using corollary 3.2.

\section{REFERENCES}

[1] J. Bertin and M. Romagny. Champs de Hurwitz. Preprint, arXiv:math/0701680v1, 2005.

[2] A. Borel. Linear algebraic groups. Graduate Texts in Mathematics, 126. Springer-Verlag, second edition, 1991.

[3] A. Cadoret. Lifting results for rational points on Hurwitz moduli spaces. Preprint, 2006, to appear in Israel J. Math.

[4] J. H. Conway, R. T. Curtis, S. P. Norton, R. A. Parker, and R. A. Wilson. Atlas of finite groups. Oxford University Press, 1985.

[5] P. Deligne and D. Mumford. The irreducibility of the space of curves of given genus. Inst. Hautes Études Sci. Publ. Math., 36:75-109, 1969.

[6] M. D. Fried and H. VölKLEIN. The inverse Galois problem and rational points on moduli spaces. Math. Ann., 290:771-800, 1991. 
[7] R. Hartshorne. Algebraic geometry. Graduate Texts in Mathematics, 52. Springer-Verlag, 1977.

[8] G. Laumon and L. Moret-Bailly. Champs algébriques. Ergebnisse der Mathematik und ihrer Grenzgebiete. 3. Folge. A Series of Modern Surveys in Mathematics, 39. SpringerVerlag, 2000.

[9] Q. LiU. Algebraic geometry and arithmetic curves. Oxford Graduate Texts in Mathematics, 6. Oxford University Press, 2002.

[10] K. Magaard, S. Shpectorov, and H. Völklein. A GAP package for braid orbit computation and applications. Experiment. Math., 12:385-393, 2003.

[11] D. Mumford and J. Fogarty. Geometric invariant theory. Ergebnisse der Mathematik und ihrer Grenzgebiete, 34. Springer-Verlag, second edition, 1982.

[12] V. Platonov and A. Rapinchuk. Algebraic groups and number theory. Pure and Applied Mathematics, 139. Academic Press Inc., 1994.

[13] J.-P. SERRE. Topics in Galois theory. Research Notes in Mathematics, 1. Jones and Bartlett Publishers, 1992.

[14] M. Suzuki. Group theory. I. Grundlehren der Mathematischen Wissenschaften, 247. Springer-Verlag, 1982.

[15] A. Tamagawa. The Grothendieck conjecture for affine curves. Compositio Math., 109:135194, 1997.

[16] S. Wewers. Construction of Hurwitz spaces. PhD thesis, IEM, Essen, 1998.

Anna Cadoret

Institut de Mathématiques de Bordeaux

Université Bordeaux 1

351 Cours de la Libération

F-33405 Talence Cedex

France

E-mail: anna.cadoret@math.u-bordeaux1.fr

Akio Tamagawa

Research Institute for Mathematical Sciences

Kyoto University

Kyoto 606-8502

Japan

E-mail: tamagawa@kurims.kyoto-u.ac.jp 